\title{
Adaptive Finite-Horizon Group Estimation for Networked Navigation Systems with Remote Sensing Complementary Observations under Mixed LOS/NLOS Environments
}

\author{
Chao Gao, ${ }^{1}$ Jianhua Lu, ${ }^{1}$ Guorong Zhao, ${ }^{1}$ and Shuang Pan $^{2}$ \\ ${ }^{1}$ Department of Control Engineering, Naval Aeronautical and Astronautical University, Yantai 264001, China \\ ${ }^{2}$ Department of Strategic Missile \& Underwater Weapon, Naval Submarine Academy, Qingdao 266071, China \\ Correspondence should be addressed to Chao Gao; gaochao.shd@163.com
}

Received 4 August 2016; Revised 25 October 2016; Accepted 26 October 2016

Academic Editor: R. Aguilar-López

Copyright (C) 2016 Chao Gao et al. This is an open access article distributed under the Creative Commons Attribution License, which permits unrestricted use, distribution, and reproduction in any medium, provided the original work is properly cited.

\begin{abstract}
Networked navigation system (NNS) enables a wealth of new applications where real-time estimation is essential. In this paper, an adaptive horizon estimator has been addressed to solve the navigational state estimation problem of NNS with the features of remote sensing complementary observations (RSOs) and mixed LOS/NLOS environments. In our approach, it is assumed that RSOs are the essential observations of the local processor but suffer from random transmission delay; a jump Markov system has been modeled with the switching parameters corresponding to LOS/NLOS errors. An adaptive finite-horizon group estimator (AFGE) has been proposed, where the horizon size can be adjusted in real time according to stochastic parameters and random delays. First, a delay-aware FIR (DFIR) estimator has been derived with observation reorganization and complementary fusion strategies. Second, an adaptive horizon group (AHG) policy has been proposed to manage the horizon size. The AFGE algorithm is thus realized by combining AHG policy and DFIR estimator. It is shown by a numerical example that the proposed AFGE has a more robust performance than the FIR estimator using constant optimal horizon size.
\end{abstract}

\section{Introduction}

Networked navigation system (NNS) [1,2], which determines its navigational state individually from the cooperative information taken with respect to spatial neighbors, has been used for a variety of purposes, such as cooperative localization [3], multivehicle cooperative guidance [4], and spatially distributed estimation [5]. NNS typically takes advantage of state estimations for accurate localization essentially based on the remote sensing observations (RSOs), which is transmitted by the cooperative NNSs through a wireless medium. Thus, during the design of navigational state estimator, several problematic issues in NNS should be considered, induced by the observation features and the transmission environment. In this research, the impact of mixed LOS/NLOS environment and complementary feature of RSOs will be investigated.

Remote sensing observations (RSOs), such as image, relative measurement, and sink knowledge, always have complementary feature, which means every observed data just comprises partial of system dynamics; thus one useful observation of estimator can only be obtained by the integration of multiple synchronous RSOs. Note that these RSOs are ubiquitous in large-scale systems [6], especially in wireless networked system (WNS) [7], and thus the state estimation based on the sensors of RSO data is brought forward. In [8], a particle filter based track-before-detect (TkBD) scheme was proposed for multiple sensors, where the sensors were asynchronous and heterogeneous; however, the TkBD schemes are computationally expensive and are mainly applicable in scene of low signal-to-noise ratios. Earlier research of RSO focused on optical sensors [9], in which the sensor noise is assumed to be Gaussian. More recent efforts focus on the radar problem, where the pixel intensity is either Rayleigh or Rician distributed depending on the absence or presence of objectives [10]. Although the extant literature considers various types of RSO sensors, 
the researches on the complementary property of wireless networks are quite few, let alone the packet-based RSO of NNS, that is, the navigational knowledge of the sender and the relative measurements.

Non-line-of-sight (NLOS) propagation might take place in a wireless network whenever there are obstacles hindering the radio signal path between the objective and its cooperative neighbors, especially in an urban region or the indoor environment. Whenever measured by received signal strength (RSS) or angle of arrival (AOA), NLOS propagation often induces transmission delay or dropout of the packets, and thus large localization errors might occur if not addressed [11]. Several strategies have been proposed for mitigating the bad effect of NLOS errors, such as statistical identification [12], hypothesis test [13], and robust parameter estimation [14]. In [15], the switching behavior of NLOS and the lineof-sight (LOS) has been described by a hidden Markov Model, and an estimator has been designed by combining Kalman filter with the interacting multiple model (IMM) approach. These results are further extended to nonlinear AOA measurement by using the MUSIC method in [16]. Similar approach has also been used to observe a mobile objective in mixed LOS/NLOS environments by using individual measurement and LOS detection [17]. However, little research has been investigated to mitigate the delay and dropout problems caused by NLOS propagation. In [18], a joint AOA and delay estimation method has been proposed for space-time coherent distributed signals based on search. The problem of joint AOA/RSS based state estimation for NNS in mixed LOS and NLOS environment has not been studied.

In reality, almost all networked systems can record the results over a recent finite horizon; for such time-varying systems, one would be more interested in the optimal estimation based on the finite recent data. When it comes to the filter design issue, finite-horizon estimation is an effective approach and gives rise to many available methods in terms of moving horizon estimation [1, 19], finite-horizon $\mathscr{H}_{\infty}$ fault estimation [20], and finite-horizon FIR estimation [21]. However, finite-horizon estimation approach has its own limitations, especially on expensively computational burden [22], with the reasons in terms of the adoption of finite recent measurements, the inversion computation [23], and the singularity problem [24]. One of the improvement methods is to manage the horizon size (window length), and several approaches [25] have been proposed. Although the existing approaches are effective with linear time-invariant systems, they can be less effective with wireless networked systems, with the reason that the constant (i.e., fixed) horizon size of the existing methods may be not able to handle the network-induced constraints owing to mixed LOS/NLOS environment. Thus, it is desirable to adapt the horizon size in finite-horizon estimation, and this motivates us to investigate this issue.

Summarizing the above analysis, it can be concluded that there is a great need to examine how the mixed LOS/NLOS environments affect the performance of the distributed estimation over a sensor network with RSO data. To address the effects of NLOS errors and random delays, a jump Markov system has been modeled with the switching parameters corresponding to LOS/NLOS errors. An adaptive finite-horizon group estimator (AFGE) has been proposed, where the horizon size can be adjusted in real time according to stochastic parameters and random delays. A numerical example is proposed to verify the effectiveness of the proposed AFGE scheme. The main contributions of this research can be highlighted as follows. (i) The observation model of NNS is a special one that has complementary property and is affected by switching LOS/NLOS errors and random transmission delay. (ii) An observation reorganization scheme and a complementary fusion strategy have been introduced to DFIR estimation procedure to mitigate the impacts of delay and complementary property, respectively. (iii) The proposed AFGE scheme contains an AHG policy, which is proposed to manage the horizon size.

The remainder of this paper is organized as follows. In Section 2, the graph-based network background is introduced, while the NNS model and some preliminaries are briefly outlined. Section 3 describes the derivation of a new DFIR estimator. Section 4 proposes the AHG method and completes the AFGE. In Section 5, an application of clustered UAVs is given for the designed AFGE. Finally, the conclusions are drawn in Section 6.

Notation. $\|u\|_{G}$ stands for the Euclidean norm of a generic vector $u ;\|u\|_{G}^{2}=u^{T} G u$, where $G$ is a symmetric positive semidefinite matrix. Given a generic vector $u_{t}, u_{t-N}^{t}=u_{t-N, t} \triangleq$ $\operatorname{col}\left(u_{t-N}, u_{t-N+1}, \ldots, u_{t}\right)$; a generic matrix $A_{t-N}^{t}=A_{t-N, t} \triangleq$ $\operatorname{col}\left(A_{t-N}, A_{t-N+1}, \ldots, A_{t}\right), t=N, N+1, \ldots ; \mathscr{L}\left\{y_{1}, \ldots, y_{n}\right\}$ denotes the linear space spanned by $y_{1}, \ldots, y_{n}$.

\section{Preliminaries and Problem Formulation}

In this section, we introduce some preliminaries related to the description of communication network and then present the problem setup.

2.1. Graph-Based Network Description. Suppose that $N_{\Sigma}$ vehicles form into an Ad hoc network, where every vehicle acts as a networked node and carries out the uniform communication protocol, that is, $[1,2,19]$. The communication topology of information flow between networked vehicles is described by a directed graph $\mathbf{G}=\left(\mathbf{C}_{g}, \mathbf{E}_{g}, \mathbf{R}_{g}\right)$, where $\mathbf{C}_{g} \in\left\{1, \ldots, N_{\Sigma}\right\}$ is the finite set of networked vehicles, $\mathbf{E}_{g} \subseteq \mathbf{C}_{g} \times \mathbf{C}_{g}$ is the set of edges, $\mathbf{R}_{g}=\left(r_{i}\right) \in \mathfrak{R}^{n}$ is the set of action radius, and $i \in \mathbf{C}_{g}$ represents the sender. The edge $e^{[i \rightarrow j]}=(i, j) \in \mathbf{E}_{g}$ indicates that node $j$ can receive the information from node $i$; that is, $(i, j) \in \mathbf{E}_{g} \Rightarrow d^{[i \rightarrow j]} \leq r_{i}$. Specifically, we define the set of neighbors as follows.

Definition 1. Let $x_{p}^{[i]} \in \mathfrak{R}^{3}$ be the position of node $\lambda \in \mathbf{C}_{g}$; the neighbor set of node $\lambda$ at time $t$ can be defined as

$$
\begin{aligned}
& \mathcal{N}_{t}^{[\lambda]}=\left\{j \in \mathbf{C}_{g}: r_{\text {inf }} \leq\left\|x_{p}^{[j]}(t)-x_{p}^{[\lambda]}(t)\right\| \leq r_{\text {sup }}, \lambda\right. \\
& \left.\quad \neq j, \mathbf{C}_{g}=\left\{1,2, \ldots, N_{\Sigma}\right\}\right\},
\end{aligned}
$$

where $r_{\text {sup }}$ and $r_{\text {inf }}$ denote the supremum and infimum radii of available communication range, respectively. Note that the 


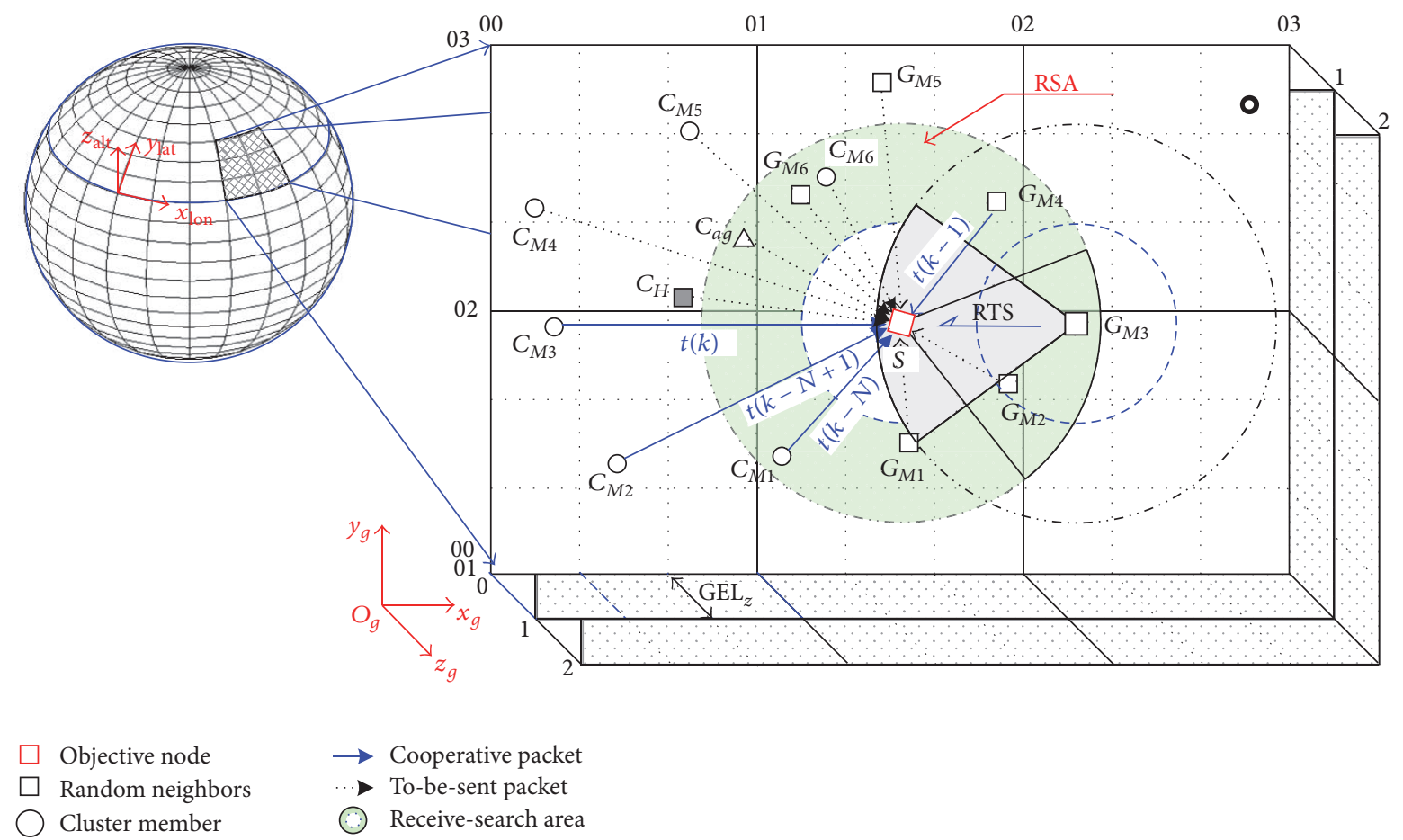

FIGURE 1: Remote sensing neighbors for networked navigation system.

relative distances between node $\lambda$ and $\mathcal{N}_{t}^{[\lambda]}$ are changeable with time $t$; thus the member of $\mathscr{N}_{t}^{[\lambda]}$ may also be changed dynamically. Besides, the effective area for cooperative neighbors can be defined by $r_{\text {sup }}$ and $r_{\text {inf }}$, namely, receive-search area (RSA), as depicted in Figure 1.

The model of RSA is defined as follows.

Definition 2. Given a generic node $\lambda \in \mathbf{C}_{g}$, its geographic neighbor $j \in \mathbf{C}_{g}$ is defined as in the RSA of node $\lambda$, if node $j$ is in the hollow sphere between $r_{\text {inf }}$ and $r_{\text {sup }}$, which are determined by RSS method as

$$
\begin{gathered}
\lg \left(r_{\text {sup }}\right)=\frac{\left(\mathrm{PL}_{\mathrm{dB}}-\gamma_{\text {sup }}\right)}{(10 \lambda)}-\frac{3.244}{\lambda}-\lg \left(f_{\mathrm{MHz}}\right), \\
\lg \left(r_{\text {inf }}\right)=\frac{\left(\mathrm{PL}_{\mathrm{dB}}-\gamma_{\text {inf }}\right)}{(10 \lambda)}-\frac{3.244}{\lambda}-\lg \left(f_{\mathrm{MHz}}\right),
\end{gathered}
$$

where $\gamma_{\text {sup }}$ and $\gamma_{\text {inf }}$ denote the received SNRs corresponding to $r_{\text {sup }}$ and $r_{\text {inf }}$, respectively, $\mathrm{PL}_{\mathrm{dB}}$ is the propagation loss $(\mathrm{dB})$ in free space propagation model [12], $\lambda$ is the path loss exponent, $2 \leq \lambda \leq 4$, and $f_{\mathrm{MHz}}$ is the electromagnetic frequency.

2.2. NNS Model and Problem Formulation. In this paper, the NNS is assumed as the essential navigation equipment of a generic vehicle $\lambda \in \mathbf{C}_{g}$. The dynamics of node $\mathcal{S}$ can be modeled as the discrete-time linear stochastic system

$$
x_{t+1}=\Phi_{t} x_{t}+\Gamma_{t} w_{t}
$$

where $x_{t}$ denotes system state; $\Phi_{t}$ and $\Gamma_{t}$ denote the system matrices which can be used to represent the motions such as the velocity model and coordinate turn model. The process noise $w_{t}$ is zero-mean white Gaussian with covariance matrix $Q_{t}$.

A set of cooperative UAVs that provide two kinds of observations, (i) navigational states of the observed neighbors, denoted by $\mathbf{x}_{t}^{\{\mathcal{N}\}}=\left\{\widetilde{x}_{t}^{[i]} \mid \widetilde{x}_{t}^{[i]}, i \in \mathcal{N}_{t}^{[\lambda]}\right\}, \widetilde{x}_{t}^{[i]}=\left(\widetilde{x}_{x, t}^{[i]}, \tilde{x}_{y, t}^{[i]}\right.$, $\tilde{x}_{z, t}^{[i]}$ ), and (ii) relative measurements (RM) between $\lambda$ and the neighbors $i \in \mathcal{N}_{t}^{[\lambda]}$, denoted by $\mathbf{z}_{t}^{\{\mathcal{W}\}}=\left\{z_{t}^{[i]} \mid z_{t}^{[i]}, i \in \mathcal{N}_{t}^{[\lambda]}\right\}$, including relative distance and relative angle, are assumed to be calculated by RSS and AOA, respectively. The detailed deductions can be found in $[12,16]$, with the basic equations as

$$
\begin{aligned}
z_{\mathrm{RSS}}^{[i]} & =\alpha-10 \lambda \lg \left|\tilde{x}^{[i]}-\widehat{x}^{[\lambda]}\right|+\eta_{\text {shad }}^{[i]}, \\
z_{\mathrm{AOA}, \theta}^{[i]} & =\arctan \left(\frac{\tilde{x}_{y}^{[i]}-\widehat{x}_{y}^{[\lambda]}}{\tilde{x}_{x}^{[i]}-\hat{x}_{x}^{[\lambda]}}\right)+\eta_{\mathrm{AOA}, \theta}^{[i]}, \\
z_{\mathrm{AOA}, \phi}^{[i]} & =\arctan \left(\frac{\tilde{x}_{z}^{[i]}-\hat{x}_{z}^{[\lambda]}}{\left\|\tilde{x}_{x, y}^{[i]}-\hat{x}_{x, y}^{[\lambda]}\right\|}\right)+\eta_{\mathrm{AOA}, \phi}^{[i]} \\
& i=1,2, \ldots, \ell .
\end{aligned}
$$

Compared to the previous DSE problems [5, 20, 25], a typical feature here is that the observations in $\mathbf{x}_{t}^{\{\mathcal{N}\}}$ and $\mathbf{z}_{t}^{\{\mathcal{N}\}}$ are mutually complementary; that is, each local observation of node $\lambda$ can only be derived through the fusion of multiple 
$x_{t}^{[i]}$ and $z_{t}^{[i]}\left(i \in \mathcal{N}_{t}^{[\lambda]}\right)$. The measure noise covariance $R_{s}^{[i]}$ in Cartesian coordinates is

$$
R_{s}^{[i]}=\phi\left(z_{\mathrm{RSS}}^{[i]}, z_{\mathrm{AOA}, \theta}^{[i]}, z_{\mathrm{AOA}, \phi}^{[i]}, \eta_{\mathrm{shad}}^{[i]}, \eta_{\mathrm{AOA}, \theta}^{[i]}, \eta_{\mathrm{AOA}, \phi}^{[i]}\right),
$$

where $z_{\mathrm{RSS}}^{[i]}, z_{\mathrm{AOA}, \theta}^{[i]}, z_{\mathrm{AOA}, \phi}^{[i]}, \eta_{\mathrm{shad}}^{[i]}, \eta_{\mathrm{AOA}, \theta}^{[i]}$, and $\eta_{\mathrm{AOA}, \phi}^{[i]}$ are defined in (4). In LOS environment, the measurements (4) are corrupted by the noise $\varepsilon_{t}^{[i]}$, which can be modeled as zeromean white Gaussian noise; that is, $\varepsilon_{t}^{[i]} \sim N\left(\varepsilon_{t}^{[i]} ; 0, R_{t, 1}^{[i]}\right)$. In NLOS environment, the measurement is corrupted by two kinds of error sources: Gaussian noise $\varepsilon_{t}^{[i]}$ and measurement bias error $e_{t}^{[i]}$. The bias error can be modeled as a biased distribution; that is, $e_{t}^{[i]} \sim N\left(e_{t}^{[i]} ; \mu_{t}^{[i]}, \Sigma_{t}^{[i]}\right)$. It is also assumed that $\varepsilon_{t}^{[i]}$ and $e_{t}^{[i]}$ are independent. Thus, the CM noise in (4) can be represented by

$$
u_{t}^{[i]}= \begin{cases}\varepsilon_{t}^{[i]}, & \text { LOS condition, } \\ \varepsilon_{t}^{[i]}+e_{t}^{[i]}, & \text { NLOS condition. }\end{cases}
$$

For the sake of notations, the measurement equation (4) can be represented in a uniform form:

$$
\begin{aligned}
f\left(\mathbf{x}_{t}^{\{\mathcal{N}\}}, \mathbf{z}_{t}^{\{\mathcal{N}\}}\right) \triangleq z_{t}^{[i]}=\phi_{t}^{[i]} x_{t}+u_{t}^{[i]}\left(\gamma_{t}^{[i]}\right) & \\
& i=1,2, \ldots, \ell,
\end{aligned}
$$

where $\phi_{t}^{[i]}$ is the nonlinear measurement function, depending on specific RM methods; the measurement noise $u_{t}^{[i]}\left(\gamma_{t}^{[i]}\right)$ is assumed to be zero-mean white Gaussian with covariance matrix $R_{t, 1}^{[i]}$ in the LOS condition; that is, $u_{t, 1}^{[i]} \in N\left(u_{t}^{[i]} ; 0, R_{t, 1}^{[i]}\right)$; and it is assumed to be white Gaussian with mean $\mu_{t}^{[i]}$ and covariance matrix $R_{t, 2}^{[i]}$ in the NLOS condition; that is, $u_{t, 2}^{[i]} \epsilon$ $N\left(u_{t}^{[i]} ; \mu_{t}^{[i]}, R_{t, 2}^{[i]}\right) \cdot \gamma_{t}^{[i]}$ is introduced to indicate the transition of LOS and NLOS measurement errors; that is, $\gamma_{t}^{[i]}=1$ represents the LOS condition and $\gamma_{t}^{[i]}=2$ represents the NLOS condition. The TPM of $\gamma_{t}^{[i]}$ is assumed to be $\Pi^{(2)}=$ $\left[\pi_{p q}^{(2)}\right]_{2 \times 2}$ with $\pi_{p q}^{(2)}=\operatorname{Pr}\left\{\gamma_{t}^{[i]}=q \mid \gamma_{t-1}^{[i]}=p\right\}$. It should be pointed out that the two-state Markov chain $\gamma_{t}^{[i]}$ is dependent on the cooperative UAV, since the objective might be in LOS condition for one UAV but in NLOS condition for another one.

Under the above mixed LOS/NLOS propagation environment, transmission delays (TDs) may decay the accuracy of relative measurement. To mitigate this disadvantage, the observed CM with TDs is modeled as

$$
\begin{array}{r}
y_{t}^{[i]}=\sum_{\tau=0}^{\hbar} \kappa_{t, \tau}^{[i]} z_{t-\tau}^{[i]}=\kappa_{t, \tau}^{[i]} \phi_{t-\tau}^{[i]} x_{t-\tau}+\kappa_{t, \tau}^{[i]} u_{t-\tau}^{[i]}\left(\gamma_{t-\tau}^{[i]}\right), \\
i=1,2, \ldots, \ell
\end{array}
$$

with $\kappa_{t, \tau}^{[i]}$ defined as a binary random variable indicating the arrival of the observation packet for state $x(t-\tau)$; that is,

$$
\kappa_{t, \tau}^{[i]}= \begin{cases}1, & \text { if } z_{t-\tau}^{[i]} \text { is received at the instant } t \\ 0, & \text { otherwise; } i \in\{1,2, \ldots, \ell\}\end{cases}
$$

Assumption 3. Random delay $\tau(t)$ is bounded with $0<$ $\tau(t) \leq \hbar$, where $\hbar$ is given as the length of memory buffer, and its probability distributions are $\operatorname{Prob}(\tau(t)=\tau)=\pi_{\tau}$, $\tau=1, \ldots, \hbar$. If the CMs transmitted to the objective with a delay larger than $\hbar$, they will be considered as the ones lost completely. Thus, the property $0 \leq \sum_{\tau=1}^{\hbar} \pi_{\tau} \leq 1$ is satisfied. Also $\tau(t)$ is independent of $x(0), w(0)$, and $u(0)$.

Obviously, $\operatorname{Prob}\left(\kappa_{t, \tau}^{[i]}=1\right)=\pi_{\tau}, \tau=1, \ldots, \hbar$. Under Assumption 3, we know that the possible received observations at time $t(t \geq \hbar)$ are

$$
\tilde{y}_{t}^{[i]}=\left[\left(\kappa_{t, 0}^{[i]} z_{t}^{[i]}\right)^{T}\left(\kappa_{t, 1}^{[i]} z_{t-1}^{[i]}\right)^{T} \cdots\left(\kappa_{t, \hbar}^{[i]} z_{t-\hbar}^{[i]}\right)^{T}\right]^{T} .
$$

In the real-time networked systems, the state $x(t)$ can only be observed at most one time, and thus $\kappa_{t, \tau}^{[i]}, \tau=1, \ldots, \hbar$, must satisfy the following property:

$$
\kappa_{t+j, j}^{[i]} \times \kappa_{t+k, k}^{[i]}=0, \quad j \neq k .
$$

When $t<\hbar$, the observation of (10) is written as

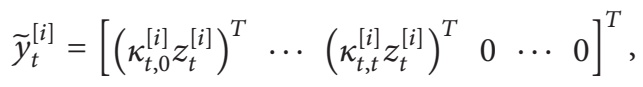

where we set $\tilde{y}_{t}^{[i]} \equiv 0$ for $t<\tau \leq \hbar$. Then the estimation problems considered in this paper can be stated as follows.

Problem $E_{t}$. For a generic node $\lambda \in \mathbf{C}_{g}$, given the set of navigation knowledge from the neighbors $\widetilde{x}_{s}^{[i]}\left(i \in \mathcal{N}_{t}^{[\lambda]}\right)$ with the stamp $s(s>0)$, the corresponding relative measures $\tilde{y}_{k}^{[i]}\left(k \in\left[t-\Xi^{[i]}, t\right]\right)$ subject to NLOS errors and random delay, with the information $\kappa_{k, \tau}^{[i]}$, find a linear minimum mean square error filter $\widehat{x}^{[\lambda]}(t \mid t)$ for navigational state of the target, $x(t)$, such that

$$
\begin{gathered}
J^{[\lambda]}=E\left\{\left\|x(t)-\widehat{x}^{[\lambda]}(t \mid t)\right\|\left|\widehat{x}_{s}^{[i]}\right|_{s \geq 0} ;\left.\tilde{y}_{k}^{[i]}\right|_{k=t-\Xi^{[i]}} ^{t} ;\right. \\
\left.\left.\kappa_{k, \tau}^{[i]}\right|_{k=t-\Xi^{[i]}} ^{t}, i \in \mathcal{N}_{t}^{[\lambda]}=\{1,2, \ldots, \ell\}\right\}
\end{gathered}
$$

is minimized, while the filter gain is stochastic.

\section{Delay-Aware FIR Estimator with Delayed Complementary Observations}

In this section, we aim to establish a unified framework to solve the addressed optimal state estimation problem in the simultaneous presence of network-induced delay and complementary property under the mixed LOS/NLOS environment, while the observations are decoded from the packets of $\ell$ spatially distributed nodes.

3.1. Delayed Observation Reorganization. In our research, the remote sensing observations (i.e., $\left\{z_{t}^{[i]}, x_{t}^{[i]}\right\}$ ) are impacted by delay and complementary issues and thus should not be adopted directly by the estimators. In this subsection, we focus on the delay issue; more specifically, we propose a preestimating scheme, including two steps, to reorganize the delay observations without dimensional augment. 
Step 1. We rearrange the complementary observations $\left\{\left.y^{[i]}(k)\right|_{t-\Xi^{[i]}} ^{t}, i \in \mathcal{N}_{t}^{[\lambda]}\right\}$, which are received in horizon $\left[t-\Xi^{[\lambda]}, t\right]$, that is, (8), and rewrite them into two parts with the batch form as

$$
\begin{gathered}
\tilde{y}_{\hbar}(s) \triangleq\left[\left.y_{0}^{[j] T}(s)\right|_{j=1} ^{\ell},\left.\quad y_{\hbar}^{[j] T}(s+\hbar)\right|_{j=1} ^{\ell}\right]^{T}, \\
t-\Xi^{[s]}-\hbar \leq s \leq t-\hbar, \\
\tilde{y}_{t-s}(s) \triangleq\left[\left.y_{0}^{[j] T}(s)\right|_{j=1} ^{\ell},\left.\quad y_{t-s}^{[j] T}(t)\right|_{j=1} ^{\ell}\right]^{T}, \\
t-\hbar<s \leq t .
\end{gathered}
$$

Then, reorganized observation $\tilde{y}(\cdot)$ is delay-free and satisfies

$$
\begin{gathered}
\tilde{y}_{\hbar}(s) \triangleq \widetilde{H}_{\hbar}(s) x(s)+\widetilde{v}_{\hbar}\left(s, \gamma_{s, s+\hbar}^{[i]}\right), \\
t-\Xi^{[s]}-\hbar \leq s \leq t-\hbar, \\
\widetilde{y}_{t-s}(s) \triangleq \widetilde{H}_{t-s}(s) x(s)+\widetilde{v}_{t-s}\left(s, \gamma_{s, t}^{[i]}\right), \quad t-\hbar<s \leq t,
\end{gathered}
$$

where

$$
\begin{aligned}
& \widetilde{H}_{\hbar}(s)=\left[\left(\left.\kappa_{s, 0}^{[i]} \phi_{s}^{[i]}\right|_{i=1} ^{\ell}\right)^{T}\left(\left.\kappa_{s+\hbar, \hbar}^{[i]} \phi_{s+h}^{[i]}\right|_{i=1} ^{\ell}\right)^{T}\right]^{T}, \\
& \widetilde{H}_{t-s}(s)=\left[\left(\left.\kappa_{s, 0}^{[i]} \phi_{s}^{[i]}\right|_{i=1} ^{\ell}\right)^{T}\left(\left.\kappa_{t, t-s}^{[i]} \phi_{t}^{[i]}\right|_{i=1} ^{\ell}\right)^{T}\right]^{T}, \\
& \widetilde{v}_{\hbar}\left(s, \gamma_{s, s+\hbar}^{[i]}\right) \\
& =\left[\left(\left.\kappa_{s, 0}^{[i]} u_{s}^{[i]}\left(\gamma_{s}^{[i]}\right)\right|_{i=1} ^{\ell}\right)^{T}\left(\left.\kappa_{s+\hbar, \hbar}^{[i]} u_{s+\hbar}^{[i]}\left(\gamma_{s+\hbar}^{[i]}\right)\right|_{i=1} ^{\ell}\right)^{T}\right]^{T}, \\
& \widetilde{v}_{t-s}\left(s, \gamma_{s, t}^{[i]}\right) \\
& =\left[\left(\left.\kappa_{s, 0}^{[i]} u_{s}^{[i]}\left(\gamma_{s}^{[i]}\right)\right|_{i=1} ^{\ell}\right)^{T}\left(\left.\kappa_{t, t-s}^{[i]} u_{t}^{[i]}\left(\gamma_{t}^{[i]}\right)\right|_{i=1} ^{\ell}\right)^{T}\right]^{T},
\end{aligned}
$$

with $v_{\hbar}(s)$ and $v_{t-s}(s)$ being white noises of zero means and covariance matrices:

$$
\begin{gathered}
\widetilde{R}_{\hbar}(s)=\operatorname{diag}\left\{\left.\kappa_{s, 0}^{[i]} R^{[i]}\right|_{i=1} ^{\ell},\left.\kappa_{s+\hbar, \hbar}^{[i]} R^{[i]}\right|_{i=1} ^{\ell}\right\}, \\
\widetilde{R}_{t-s}(s)=\operatorname{diag}\left\{\left.\kappa_{s, 0}^{[i]} R^{[i]}\right|_{i=1} ^{\ell},\left.\kappa_{s+\hbar, \hbar}^{[i]} R^{[i]}\right|_{i=1} ^{\ell}\right\} .
\end{gathered}
$$

Moreover, we can follow the result in [1] that the following lemma is true.

Lemma 4. For the given time horizon $\left[t-\Xi^{[\lambda]}, t\right]$, the linear space $\mathscr{L}_{1} \triangleq\left\{\{y(k)\}_{k=t-\Xi^{[\lambda]}}^{t}\right\}$ generated by $\{y(k)\}_{k=t-\Xi^{[\lambda]}}^{t}$ is equivalent to the linear space of

$$
\mathscr{L}_{2} \triangleq\left\{\left\{\tilde{y}_{\hbar}(s)\right\}_{s=t-\Xi^{[\lambda]}-\hbar}^{t-\hbar-1} ;\left\{\tilde{y}_{t-s}(s)\right\}_{s=t-\hbar}^{t}\right\}
$$

which implies that $\mathscr{L}_{2}$ contains the same information as $\mathscr{L}_{1}$.
Step 2. We further fuse the complementary observations (15) of the same stamp $s \in\left[t-\Xi^{[\lambda]}-\hbar, t\right)$ into one complete measurement $\tilde{y}_{m}^{[\lambda]}(s)(m \in\{\hbar, t-s\})$ prior to the local estimation process, and thus

$$
\begin{aligned}
\tilde{y}_{m}^{[\lambda]}(s)=\bar{R} \cdot \sum_{i=1}^{\ell} g\left(\tilde{y}_{m}^{[i]}(s), x_{m}^{[i]}(s)\right), \\
\quad m \in\{h, t-s\}, s=t-\Xi^{[\lambda]}-\hbar, \ldots, t,
\end{aligned}
$$

where $\bar{R}$ denotes the weighted matrix; $g\left(\tilde{y}_{m}^{[i]}(s), x_{m}^{[i]}(s)\right)$ is the relative measure function. Thus, the local measurements $\tilde{y}_{m}^{[\lambda]}(s) \in \mathfrak{R}^{n_{y}}$ in (19) can be rewritten in the reorganized linear space $\mathscr{L}_{2}$ as

$$
\tilde{y}_{m}^{[\lambda]}(s)=\vartheta_{s}^{[\lambda]} \widetilde{H}_{m}^{[\lambda]}(s) x(s)+\widetilde{v}_{m}^{[\lambda]}\left(s, \gamma_{m}^{[\lambda]}\right),
$$

where $\vartheta_{s}^{[\lambda]}$ represents the failed fusion of $\widetilde{y}^{[\lambda]}(s)$ at time stamp $s=t-\Xi^{[\lambda]}-\hbar, \ldots, t-1$, which takes the value of 0 or 1 , and is modeled as Bernoulli distributed white sequences satisfying $\operatorname{Prob}\left\{\vartheta_{s}^{[\lambda]}=1\right\}=\bar{\vartheta}^{[\lambda]} ; 0<\bar{\vartheta}^{[\lambda]} \leq 1$.

Remark 5. Note that $\vartheta_{s}^{[\lambda]}\left(s=t-\Xi^{[\lambda]}-\hbar, \ldots, t-1\right)$ is introduced to represent the failure of the preestimating algorithm (19) at time $s$, with the reason that the numbers of reorganized CMs $\tilde{y}_{\hbar \mid t}(s)$ or $\tilde{y}_{t-s \mid t}(s)$ are less than a lower limit $[9,12]$. Thus, through preestimating transformation, the remote sensing CMs in (14) with high dimension are transformed into the data missing in (20) with lower dimension.

Besides, $\bar{R}$ and $g\left(\widetilde{y}_{m}^{[i]}(s), x_{m}^{[i]}(s)\right)$ in (19) will be deduced through a complementary fusion strategy, with the details as follows.

3.2. Complementary Fusion Strategy. Let $\left\{\widetilde{y}_{m}^{[i]}(s), i=1,2, \ldots\right.$, $\ell$ \} be the set of complementary observations generated at instant $s$, the relative measures between objective $\lambda$ and its neighbors $i \in \mathcal{N}_{s}^{[\lambda]}$, that is, relative distance and relative angle, are calculated through RSS and AOA, respectively, as depicted in (4), and the measure noise covariance $\widetilde{R}_{m}^{[i]}(s)$ in (17).

Hereafter, the fused measurement covariance $\bar{R}$ and the fused measurement variable $y_{m}^{[\lambda]}(s)(m \in\{\hbar, t-s\})$ can be expressed as

$$
\begin{aligned}
\bar{R}= & \left(\sum_{i=1}^{\ell}\left(\widetilde{R}_{m}^{[i]}(s)\right)^{-1}\right)^{-1}, m \in\{\hbar, t-s\} \\
y_{m}^{[\lambda]}(s) & \bar{R} \\
= & \quad \sum_{i=1}^{\ell}\left(\left(\widetilde{R}_{m}^{[i]}(s)\right)^{-1}\left(\widetilde{y}_{m}^{[i]}(s)+x_{m}^{[\ell]}(s)-x_{m}^{[i]}(s)\right)\right) .
\end{aligned}
$$

Besides, the sensitivity matrix of the fused measurement variable (22) can be written as

$$
\bar{H}=\bar{R} \cdot \sum_{i=1}^{\ell}\left[\left(\widetilde{R}_{m}^{[i]}(s)\right)^{-1} H^{[i]}\right] ;
$$


hereinto, $H^{[i]}$ is the sensitivity matrix corresponding to $\tilde{y}_{m}^{[i]}(s)$ :

$$
H^{[i]}=\left[\begin{array}{ll}
-I_{3 \times 3} & \mathbf{0}_{3 \times 3}
\end{array}\right] .
$$

Note that the estimation error in this research may not always fall within the confidence level of the predicted covariance boundary. To avoid this problematic scenario, we introduce a new weighting factor $\psi_{i, y}$ as follows.

Definition 6. Given the CMs $\tilde{y}_{m}^{[i]}(s), m \in\{\hbar, t-s\}$, sensitivity matrix $H^{[i]}$, and the inverse measurement noise variance, $\left(\widetilde{R}_{m}^{[i]}(s)\right)^{-1}$, the weighting factor, $\psi_{i, y}$, is applied before they are fused; more specifically, it is defined as

$$
\psi_{i, y}=\frac{\varrho_{i}^{2}}{\varrho^{T} \varrho},
$$

where $\sum_{i=1}^{\ell} \psi_{i, y}=1 ; \varrho=\left[\begin{array}{llll}\varrho_{1} & \varrho_{2} & \cdots & \varrho_{\ell}\end{array}\right]^{T}$.

The weighting factor $\psi_{i, y}$ is a function of (4). For instance, RSS vector is defined as $\mathscr{P}_{m, \mathrm{rss}}=\left[\begin{array}{lll}\tilde{y}_{m, \mathrm{rss}}^{[1]} & \cdots & \tilde{y}_{m, \mathrm{rss}}^{[\ell]}\end{array}\right]^{T}, m \in$ $\{\hbar, t-s\}$. In the proposed weight strategy, the weighting factor of each CM is assigned based on the relative measures between $i \in \mathcal{N}_{s}^{[\lambda]}$ and $\lambda$. Lower weight is assigned to the neighbors $i \in \mathcal{N}_{s}^{[\lambda]}$ closer to $\lambda$. In other words, weighting factor $\psi_{i, y}$ is selected to be proportional to the relative measures between the neighbors. The last measure $\tilde{y}_{m, \mathrm{rss}}^{[\ell]}$, which is equivalent to $\max \left(\mathscr{P}_{m, \mathrm{rss}}\right)$, has highest $\psi_{i, y}$. Define the terms $\Delta \mathscr{P}_{m, \text { rss }}$ and $\varrho$ as

$$
\begin{aligned}
\Delta \mathscr{P}_{m, \mathrm{rss}}^{[i]} & =\tilde{y}_{m, \mathrm{rss}}^{[\ell]}-\tilde{y}_{m, \mathrm{rss}}^{[i]}, \quad m \in\{\hbar, t-s\}, \\
\varrho_{i} & =1-\frac{\Delta \mathscr{P}_{m, \mathrm{rss}}^{[i]}}{\left\|\Delta \mathscr{P}_{m, \mathrm{rss}}^{[i]}\right\|}, \quad m \in\{\hbar, t-s\},
\end{aligned}
$$

where $\Delta \mathscr{P}_{m, \mathrm{rss}}=\left[\Delta \mathscr{P}_{m, \mathrm{rss}}^{[1]}, \Delta \mathscr{P}_{m, \mathrm{rss}}^{[2]}, \ldots, \Delta \mathscr{P}_{m, \mathrm{rss}}^{[\ell]}\right]$.

Thus, the newly fused measurement noise covariance in (21) can be rewritten as

$$
\bar{R}=\ell \cdot\left(\sum_{i=1}^{\ell} \psi_{i, y}\left(\widetilde{R}_{m}^{[i]}(s)\right)^{-1}\right)^{-1}, \quad m \in\{\hbar, t-s\}
$$

Furthermore, the sensitivity matrix in (23) can be rewritten as

$$
\begin{aligned}
\bar{H}= & \left(\sum_{i=1}^{\ell} \psi_{i, y}\left(\widetilde{R}_{m}^{[i]}(s)\right)^{-1}\right)^{-1} \\
& \cdot \sum_{i=1}^{\ell}\left[\psi_{i, y}\left(\widetilde{R}_{m}^{[i]}(s)\right)^{-1} H^{[i]}\right], \quad m \in\{\hbar, t-s\} .
\end{aligned}
$$

Meanwhile, the newly fused measurement vector in (22) can be rewritten as

$$
\begin{aligned}
& y_{m}^{[\lambda]}(s)=\bar{R} \cdot \sum_{i=1}^{\ell} g\left(\tilde{y}_{m}^{[i]}(s), x_{m}^{[i]}(s)\right)=\{\ell \\
& \left.\cdot\left(\sum_{i=1}^{\ell} \psi_{i, y}\left(\widetilde{R}_{m}^{[i]}(s)\right)^{-1}\right)^{-1}\right\} \sum_{i=1}^{\ell}\left\{\frac{1}{\ell}\right. \\
& \left.\cdot\left[\psi_{i, y}\left(\widetilde{R}_{m}^{[i]}(s)\right)^{-1}\left(\widetilde{y}_{m}^{[i]}(s)+x_{m}^{[\ell]}(s)-x_{m}^{[i]}(s)\right)\right]\right\}, \\
& m \in\{\hbar, t-s\},
\end{aligned}
$$

where

$$
\begin{aligned}
g & \left(\tilde{y}_{m}^{[i]}(s), x_{m}^{[i]}(s)\right) \\
= & \frac{1}{\ell} \\
& \cdot\left[\psi_{i, y}\left(\widetilde{R}_{m}^{[i]}(s)\right)^{-1}\left(\tilde{y}_{m}^{[i]}(s)+x_{m}^{[\ell]}(s)-x_{m}^{[i]}(s)\right)\right], \\
& m \in\{\hbar, t-s\} .
\end{aligned}
$$

Note that the resulting problem is solved by a robust recursive scheme, which allows one to determine approximate estimates with a reduced computational condition. The details are presented in the following subsection.

3.3. Delay-Aware FIR Estimation Algorithm. In this subsection, we proposed a novel unbiased FIR estimator, namely, delay-aware FIR (DFIR) estimation algorithm, to realize the network-based state estimation of a generic node $\lambda \epsilon$ $\mathrm{C}_{g}$ based on the cooperative observation of its neighborhood $\mathcal{N}_{s}^{[\lambda]}$. Note that several unique network-induced constraints are involved, including complementary observations, Markov jump parameters, and random delay; hereinto, partial constraints have been transformed in Sections 3.1 and 3.2, which may simplify the design procedure of DFIR.

Prior to the main result of this section, let us recall a useful lemma that will be useful in DFIR algorithm.

Lemma 7 (see [24]). Suppose that the general trace optimization (GTO) problem is given as

$$
\min _{\overline{\mathscr{H}}} \operatorname{tr}\left[(\overline{\mathscr{H}} \mathscr{A}-\mathscr{B}) \mathscr{C}(\overline{\mathscr{H}} \mathscr{A}-\mathscr{B})^{T}+\overline{\mathscr{H}} \mathscr{D} \overline{\mathscr{H}}^{T}\right],
$$

subject to $\overline{\mathscr{H}}^{\mathscr{E}}=\mathscr{F}$,

where $\mathscr{A}, \mathscr{B}, \mathscr{C}, \mathscr{D}, \mathscr{E}$, and $\mathscr{F}$ denote the constant matrices with appropriate dimensions: $\mathscr{C}=\mathscr{C}^{T}>0$ and $\mathscr{D}=\mathscr{D}^{T}>0$. $\operatorname{tr}(\mathscr{F})$ is the trace of the main diagonal of $\mathscr{J}$.

Thus, the unique solution to the GTO problem can be obtained as

$$
=\left[\begin{array}{ll}
\mathscr{F} & \mathscr{B}
\end{array}\right]\left[\begin{array}{c}
\left(\mathscr{E}^{T} \Lambda^{-1} \mathscr{E}\right)^{-1} \mathscr{E}^{T} \Lambda^{-1} \\
\mathscr{C} \mathscr{A}^{T} \Lambda^{-1}\left[I-\mathscr{E}\left(\mathscr{E}^{T} \Lambda^{-1} \mathscr{E}\right)^{-1} \mathscr{E}^{T} \Lambda^{-1}\right]
\end{array}\right],
$$

where $\Lambda=\mathscr{A} \mathscr{C} \mathscr{A}^{T}+\mathscr{D}$. 
On the recent finite-time horizon $\left[t-\Xi^{[\lambda]}-\hbar, t-1\right]$ of reorganized linear space $\mathscr{L}_{2}$, the receding horizon filter for (3) and (20) can be written as

$$
\hat{x}_{t \mid t-1}^{[\lambda]}=\sum_{k=t-\Xi^{[\lambda]}-\hbar}^{t-1} \widetilde{H}_{t-k, \gamma(s)} \tilde{y}_{\hbar}^{[\lambda]}(s)=\widetilde{\mathscr{H}}_{\gamma(s)} \widetilde{\mathscr{Y}}_{t-1}^{[\lambda]},
$$

where $\widetilde{\mathscr{H}}_{\gamma(s)}$ is the gain matrix, $\widetilde{\mathscr{Y}}_{t-1}^{[\lambda]}$ is the augmented measurement vector, and $\gamma(s)$ is the set of switching factors on the horizon $\left[t-\Xi^{[\lambda]}-\hbar, t-1\right]$ defined by (34)-(36), respectively, as

$$
\widetilde{\mathscr{H}}_{\gamma(s)} \triangleq\left[\begin{array}{llll}
\widetilde{H}_{\Xi^{[\lambda]}+\hbar, \gamma(s)} & \widetilde{H}_{\Xi^{[\lambda]}+\hbar-1, \gamma(s)} & \cdots & \widetilde{H}_{1, \gamma(s)}
\end{array}\right],
$$

$$
\begin{aligned}
& \widetilde{\mathscr{Y}}_{t-1}^{[\lambda]} \triangleq\left[\begin{array}{llll}
\tilde{y}_{t-\Xi^{[\lambda]}-\hbar}^{[\lambda] T} & \tilde{y}_{t-\Xi^{[\lambda]}-\hbar+1}^{[\lambda] T} & \cdots & \tilde{y}_{t-1}^{[\lambda] T}
\end{array}\right]^{T},
\end{aligned}
$$

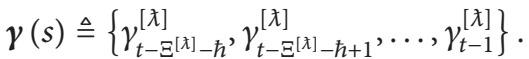

For ease of notation only, we express the horizon size of estimator $\Xi^{[\lambda]}+\hbar$ as $\widetilde{\Xi}$; that is, $\widetilde{\Xi} \triangleq \Xi^{[\lambda]}+\hbar, \widetilde{\mathscr{Y}}_{t-1}^{[\lambda]}$ can be rewritten in the batch form as

$$
\widetilde{\mathscr{Y}}_{t-1}^{[\lambda]}=\mathscr{F}_{\widetilde{\Xi}, \gamma(s)} x_{t-\widetilde{\Xi}}+\mathscr{H}_{\widetilde{\Xi}, \gamma(s)} \mathscr{W}_{t-1}+\mathscr{V}_{t-1, \gamma(s)},
$$

where

$$
\begin{aligned}
& \mathscr{F}_{\widetilde{\Xi}, \gamma(s)} \triangleq\left[\begin{array}{c}
\widetilde{H}_{t-\widetilde{\Xi},[\}_{t-\Xi}^{[\lambda]}} F_{t-\widetilde{\Xi}}^{t-1} \\
\vdots \\
\widetilde{H}_{t-\widetilde{\Xi}+1, \gamma_{t-\widetilde{\Xi}+1}^{[\lambda]}} F_{t-2}^{t-1} \\
\widetilde{H}_{t-1, \gamma_{t-1}^{[]}} \Phi_{t-1}^{-1}
\end{array}\right] \\
& \mathscr{W}_{t-1} \triangleq\left[\begin{array}{c}
w_{t-\tilde{\Xi}} \\
w_{t-\tilde{\Xi}+1} \\
\vdots \\
w_{t-1}
\end{array}\right], \\
& \mathscr{V}_{t-1, \gamma(s)} \triangleq\left[\begin{array}{c}
\widetilde{v}_{t-\widetilde{\Xi}, \gamma_{t-\tilde{\Xi}}^{[\lambda]}} \\
\widetilde{v}_{t-\widetilde{\Xi}+1,\left[{ }_{t-\tilde{\Xi}+1}^{[\lambda]}\right.} \\
\vdots \\
\widetilde{v}_{t-1, \gamma_{t-1}^{[\lambda]}}
\end{array}\right]
\end{aligned}
$$

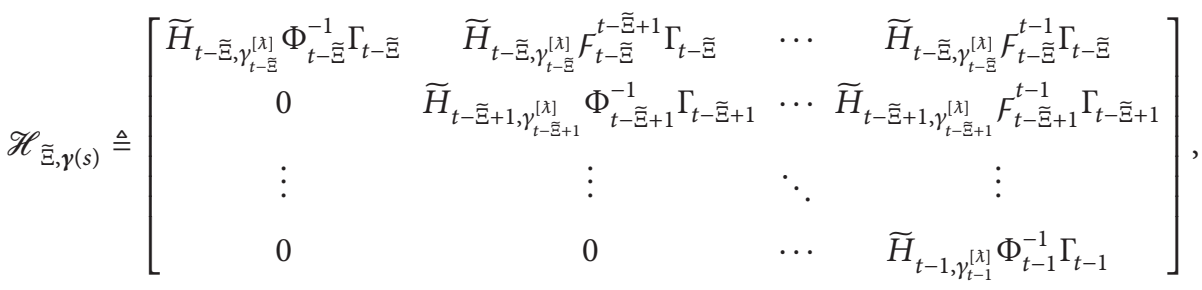

$$
\begin{aligned}
& Q_{\widetilde{\Xi}} \triangleq[\operatorname{diag}(\overbrace{Q, Q, \ldots, Q}^{\widetilde{\Xi}})], \\
& \mathscr{R}_{\widetilde{\Xi}} \triangleq\left[\operatorname{diag}\left(\widetilde{\widetilde{R}}_{\widetilde{R}, \ldots, \widetilde{R}}^{\widetilde{\Xi}}\right)\right], \\
& F_{t-g}^{t-h} \triangleq \prod_{i=g}^{h} \Phi_{t-i}^{-1}=\Phi_{t-g}^{-1} \Phi_{t-g+1}^{-1} \cdots \Phi_{t-h}^{-1} .
\end{aligned}
$$


The noise terms $\mathscr{H}_{\widetilde{\Xi}, \gamma(s)} \mathscr{W}_{t-1}+\mathscr{V}_{t-1, \gamma(s)}$ in (37) can be expressed as zero mean with covariance $\Theta_{\widetilde{\Xi}, \gamma(s)}$ by

$$
\begin{aligned}
\Theta_{i, \gamma(s)}= & \mathscr{H}_{i, \gamma(s)}[\operatorname{diag}(\overbrace{\mathrm{Q}, Q, \ldots, Q}^{i})] \mathscr{H}_{i, \gamma(s)}^{T} \\
& +[\operatorname{diag}(\overbrace{\widetilde{R}, \widetilde{R}, \ldots, \widetilde{R}}^{i})] .
\end{aligned}
$$

Substituting (37) into (33) and taking the expectation on both sides of (33), the following relations can be obtained:

$$
\begin{aligned}
& \widehat{x}_{t \mid t-1}=\overline{\mathscr{H}} \widetilde{\mathscr{Y}}_{t-1}^{[\lambda]} \\
& =\overline{\mathscr{H}} \mathscr{F}_{\widetilde{\Xi}, \gamma(s)} x_{t-\widetilde{\Xi}} \\
& +\overline{\mathscr{H}}\left(\mathscr{H}_{\widetilde{\Xi}, \gamma(s)} \mathscr{W}_{t-1}+\mathscr{V}_{t-1, \gamma(s)}\right), \\
& E\left[\widehat{x}_{t \mid t-1}\right]=\overline{\mathscr{H}} \mathscr{F}_{\widetilde{\Xi}, \gamma(s)} E\left[x_{t-\widetilde{\Xi}}\right],
\end{aligned}
$$

with the horizon initial conditions

$$
\begin{aligned}
& \widehat{x}_{t-\widetilde{\Xi}}=\omega_{t-\widetilde{\Xi}}=E\left[x_{t-\widetilde{\Xi}}\right], \\
& \widetilde{P}_{t-\widetilde{\Xi}}=\widetilde{P}_{t-\widetilde{\Xi}}=\operatorname{cov}\left\{x_{t-\widetilde{\Xi}}, x_{t-\widetilde{\Xi}}\right\}
\end{aligned}
$$

determined by Lyapunov equation on the horizon $k \in$ $\left[t_{0}, t_{t-\widetilde{\Xi}}\right)$ as

$$
\begin{aligned}
\omega_{k+1} & =\Phi_{k} \omega_{k}, \\
\omega_{t_{0}} & =\omega_{0}, \\
\widetilde{P}_{k+1} & =\Phi_{k} \widetilde{P}_{k}+\Phi_{k}^{T}+\widetilde{Q}_{k}, \\
\widetilde{P}_{t_{0}} & =\widetilde{P}_{0} .
\end{aligned}
$$

Consider the unbiased condition; that is, $E\left[\widehat{x}_{t \mid t-1}\right]=$ $E\left[x_{t-\tilde{\Xi}}\right]$, and, irrespective of the initial states, constraint (43) is required:

$$
\overline{\mathscr{H}} \mathscr{F}_{\widetilde{\Xi}, \gamma(s)}=I .
$$

Thus, the objective now turns to obtain the optimal gain matrix $\overline{\mathscr{H}}^{\circ}$ with the unbiasedness constraint (43), such that the estimation error $e_{t}$ of the estimate $\widehat{x}_{t \mid t-1}$ has the following minimum variance:

$$
\begin{aligned}
\overline{\mathscr{H}}^{O} & =\arg \min _{\overline{\mathscr{H}}} E\left[e_{t}^{T} e_{t}\right] \\
& =\arg \min _{\overline{\mathscr{H}}} E\left\{\operatorname{tr}\left[x_{t}-\widehat{x}_{t \mid t-1}\right]^{T}\left[x_{t}-\widehat{x}_{t \mid t-1}\right]\right\} \\
& =\arg \min _{\overline{\mathscr{H}}}\left(\mathscr{H}_{\widetilde{\Xi}, \gamma(s)} \mathscr{W}_{t-1}+\mathscr{V}_{t-1, \gamma(s)}\right)^{T} \\
& \overline{\mathscr{H}}^{T} \overline{\mathscr{H}}\left(\mathscr{H}_{\widetilde{\Xi}, \gamma(s)} \mathscr{W}_{t-1}+\mathscr{V}_{t-1, \gamma(s)}\right) .
\end{aligned}
$$

By using Lemma 7, the gain matrix $\widetilde{\mathscr{H}}$ can be obtained by the following correspondences:

$$
\begin{aligned}
& \mathscr{A} \longleftarrow \mathscr{H}_{\widetilde{\Xi}}, \\
& \mathscr{B} \longleftarrow O, \\
& \mathscr{C} \longleftarrow \mathscr{Q}_{\widetilde{\Xi}}, \\
& \mathscr{D} \longleftarrow \mathscr{R}_{\widetilde{\Xi}}, \\
& \mathscr{E} \longleftarrow \mathscr{F}_{\widetilde{\Xi}, \gamma(s)}, \\
& \mathscr{F} \longleftarrow I .
\end{aligned}
$$

Thus, when $\left\{\Phi_{k}, \widetilde{H}_{k, \gamma_{k}^{[\lambda]}}\right\}(k \in[t-\widetilde{\Xi}, t-1])$ is observable and $\Xi^{[\lambda]}+\hbar \geq n$, the DFIR for the reorganized system (20) can be given as

$$
\begin{aligned}
& \widehat{x}_{t \mid t-1}^{[\lambda]}=\overline{\mathscr{H}}^{0} \widetilde{\mathscr{Y}}_{t-1}^{[\lambda]} \\
& =\left[\begin{array}{ll}
I & 0
\end{array}\right]\left[\begin{array}{c}
\left(\mathscr{F}_{\widetilde{\Xi}, \gamma(s)}^{T} \Theta_{\widetilde{\Xi}, \gamma(s)}^{-1} \mathscr{F}_{\widetilde{\Xi}, \gamma(s)}\right)^{-1} \mathscr{F}_{\widetilde{\Xi}, \gamma(s)}^{T} \Theta_{\widetilde{\Xi}, \gamma(s)}^{-1} \\
*
\end{array}\right] \widetilde{\mathscr{Y}}_{t-1}^{[\lambda]} \\
& =\left(\mathscr{F}_{\widetilde{\Xi}, \gamma(s)}^{T} \Theta_{\widetilde{\Xi}, \gamma(s)}^{-1} \mathscr{F}_{\widetilde{\Xi}, \gamma(s)}\right)^{-1} \mathscr{F}_{\widetilde{\Xi}, \gamma(s)}^{T} \Theta_{\widetilde{\Xi}, \gamma(s)}^{-1} \widetilde{\mathscr{Y}}_{t-1}^{[\lambda]} .
\end{aligned}
$$

Remark 8. The batch form of RHE (44) requires the inversion computation of matrices $\Theta_{\widetilde{\Xi}, \gamma(s)}^{-1}$ and $\mathscr{F}_{\widetilde{\Xi}, \gamma(s)}^{T} \Theta_{\widetilde{\Xi}, \gamma(s)}^{-1} \mathscr{F}_{\widetilde{\Xi}, \gamma(s)}$, whose dimension will become large as the horizon length $\Xi^{[\chi]}$ increases; besides, the singularity problem will be encountered due to the impacts of LOS/NLOS condition.

Remark 9. The FIR filter uses finite measurements of the recent time interval. The length of the interval is called horizon size, which is also referred to as the window size or memory size. In (13), the matrices with subscript " $\Xi^{[\lambda] \text { ” }}$ are obtained using the information on the recent time interval $\left[t-\Xi^{[\lambda]}-\hbar, t-1\right]$, where the horizon size is $\Xi^{[\lambda]}+\hbar$. The large matrices defined in (13) are time-varying.

\section{Adaptive Finite-Horizon Group Estimator}

In this section, we introduce an adaptive horizon group (AHG) approach to manage the horizon size in DFIR estimator. Different from the previous horizon group shift policy [26], AHG policy is designed to adjust the horizon size of DFIR algorithm according to the dynamic changed cooperative neighbors and the concerned network constraints. The AHG algorithm was combined with the proposed DFIR estimator and obtained AFGE estimator. The detailed algorithm of estimating with AHG is presented below.

The first step of estimation with the DFIR is to construct a group of horizon sizes (i.e., horizon group). The horizon group at time $s$ is denoted by $\mathscr{G}_{s}$ and is defined as

$$
\begin{aligned}
\mathscr{G}_{s} & =\left\{\widetilde{\Xi} \mid \widetilde{\Xi}=2 \Xi_{s}^{c}+\left(\zeta \times \tau_{s}^{[i]}-\hbar\right), \tau_{s}^{[i]}\right. \\
& =0,1,2, \ldots, \hbar\},
\end{aligned}
$$




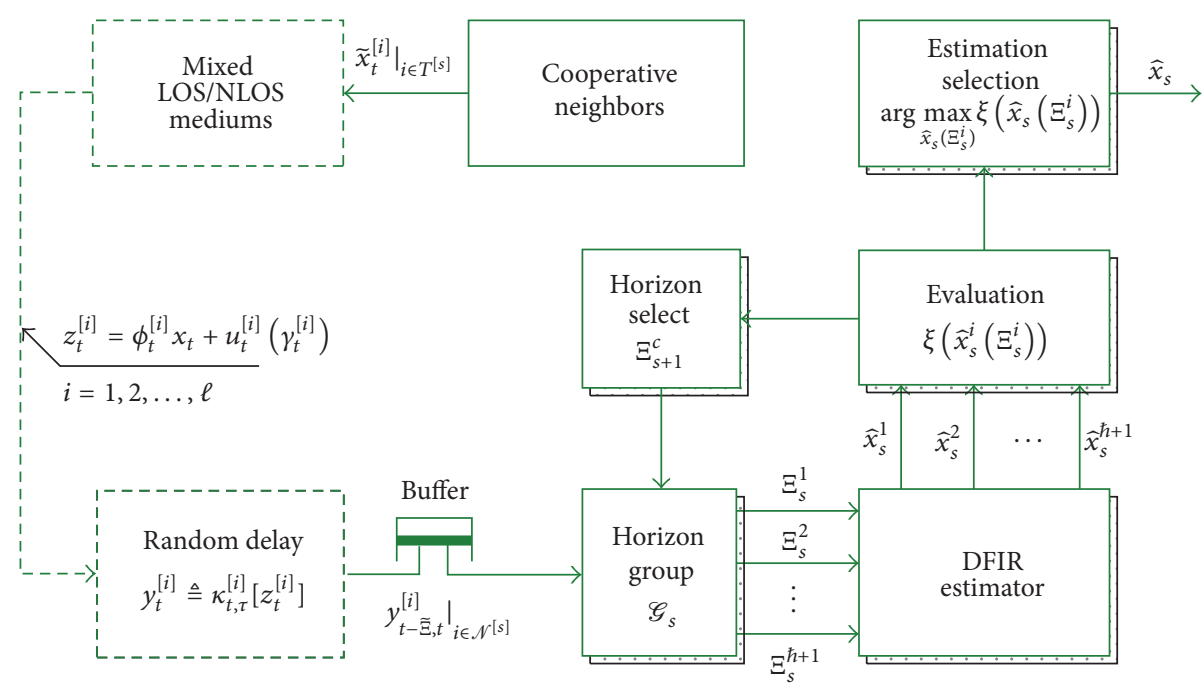

FIGURE 2: Adaptive finite-horizon group estimation scheme with AHG.

where $\widetilde{\Xi}$ is the horizon size, $\Xi_{s}^{c}$ is the center horizon size at time $s, \zeta$ is the interval between horizon sizes, and $\hbar+1$ is the total number of horizon sizes in a horizon group. Note that $\tau_{s}^{[i]}$ impliedly reflected the possible transmission delay from the cooperative neighbor $i \in \mathcal{N}_{s}^{[\lambda]}$, and $\hbar$ denotes the tolerable maximum transmission delay, which is defined in Assumption 3.

The second step is to execute the DFIR estimator repeatedly with the horizon sizes in $\mathscr{G}_{s}$, which produces a group of state estimates, $\hat{x}_{s}^{1}, \hat{x}_{s}^{2}, \ldots, \hat{x}_{s}^{\hbar+1}$. The reliable estimate is then selected by maximizing the likelihood function of $y_{m}^{[\lambda]}(s) \quad g_{Y}\left(y_{m}^{[\lambda]}(s) \mid x=\hat{x}_{s}(\widetilde{\Xi})\right)$, where $\hat{x}_{s}(\widetilde{\Xi})$ is the state estimate obtained from (46) with the horizon size $\widetilde{\Xi}$. Note that the density function of $\widetilde{v}_{m}^{[\lambda]}\left(s, \gamma_{m}^{[\lambda]}\right)$ is $g_{V}\left(\widetilde{v}_{m}^{[\lambda]}\left(s, \gamma_{m}^{[\lambda]}\right)\right)$, where

$$
\begin{aligned}
& g_{V}\left(\widetilde{v}_{m}^{[\lambda]}\left(s, \gamma_{m}^{[\lambda]}\right)\right)=\frac{1}{\sqrt{(2 \pi)^{q} \operatorname{det}(\bar{R})}} \\
& \cdot \exp \left\{-\frac{1}{2}\left(\tilde{v}_{m}^{[\lambda]}\left(s, \gamma_{m}^{[\lambda]}\right)\right)^{T} \bar{R}^{-1} \widetilde{v}_{m}^{[\lambda]}\left(s, \gamma_{m}^{[\lambda]}\right)\right\} .
\end{aligned}
$$

The state estimates are then evaluated based on their likelihoods, which can be computed as follows:

$$
\begin{aligned}
& \xi\left(\widehat{x}_{s}^{i}(\widetilde{\Xi})\right)=p\left(y_{m}^{[\lambda]}(s) \mid \widehat{x}_{s}^{i}(\widetilde{\Xi})\right)=\frac{1}{\sqrt{(2 \pi)^{q} \operatorname{det}(\bar{R})}} \\
& \quad \times \exp \left\{-\frac{1}{2}\left(y_{m}^{[\lambda]}(s)-\vartheta_{s}^{[\lambda]} \widetilde{H}_{m}^{[\lambda]}(s) \hat{x}_{s}^{i}(\widetilde{\Xi})\right)^{T}\right. \\
& \left.\cdot \bar{R}^{-1}\left(y_{m}^{[\lambda]}(s)-\vartheta_{s}^{[\lambda]} \widetilde{H}_{m}^{[\lambda]}(s) \hat{x}_{s}^{i}(\widetilde{\Xi})\right)\right\},
\end{aligned}
$$

where $\xi\left(\widehat{x}_{s}^{i}(\widetilde{\Xi})\right)$ is the likelihood of the state estimate $\widehat{x}_{s}^{i}(\widetilde{\Xi})$ and $\widetilde{H}_{m}^{[\lambda]}(s)$ is the measurement matrix defined in (20):

$$
\begin{gathered}
\mathscr{J}\left(\widehat{x}_{s}(\widetilde{\Xi})\right)=\frac{1}{2}\left[\left(y_{m}^{[\lambda]}(s)-\vartheta_{s}^{[\lambda]} \widetilde{H}_{m}^{[\lambda]}(s) \hat{x}_{s}^{i}(\widetilde{\Xi})\right)^{T}\right. \\
\left.\cdot \bar{R}^{-1}\left(y_{m}^{[\lambda]}(s)-\vartheta_{s}^{[\lambda]} \widetilde{H}_{m}^{[\lambda]}(s) \hat{x}_{s}^{i}(\widetilde{\Xi})\right)\right] .
\end{gathered}
$$

Thus, a reliable estimate is selected by minimizing $\mathscr{J}\left(\widehat{x}_{s}(\widetilde{\Xi})\right)$. We use $\mathscr{J}\left(\widehat{x}_{s}(\widetilde{\Xi})\right)$ as a measure of accuracy of the estimation result. The smaller the cost $\mathscr{J}\left(\widehat{x}_{s}(\widetilde{\Xi})\right)$ is, the more reliable the estimated state is.

The final step is to change the horizon group, which is called the AHG. If we determine $\Xi_{s}^{c}, \mathscr{G}_{s}$ is determined automatically using (47). The horizon size that produces a reliable estimate at time $s$ is used as the center horizon size at time $s+1$. Let $\widehat{x}_{s}\left(\Xi_{s}^{i}\right)$ be the state estimate obtained from the FIR estimator using the horizon size $\Xi_{s}^{i}$. The AHG can then be described by the following equation:

$$
\Xi_{s+1}^{c}=\arg \max _{\Xi_{s}^{c}} \xi\left(\widehat{x}_{s}\left(\Xi_{s}^{i}\right)\right)
$$

In this way, the horizon group is moved (or shifted) toward the horizon size that produces good estimation performance. In the AHG algorithm, the horizon size varies over time. Thus, we impose the lower bound $\widetilde{\Xi}_{\min }$ and the upper bound $\widetilde{\Xi}_{\max }$ on the horizon size. We now summarize the estimation algorithm with DFIR, as depicted in Figure 2, whereas the detailed algorithm is presented as follows.

Algorithm 10 (AFGE estimator). Select design parameters of the AHG algorithm, such as $\hbar, \zeta, \widetilde{\Xi}_{\min }$, and $\widetilde{\Xi}_{\text {max }}$. Set the initial 
value of the center horizon size as $\Xi_{s}^{c}=\widetilde{\Xi}_{\min }$. For $s=\widetilde{\Xi}_{\min }+$ $1, \widetilde{\Xi}_{\min }+2, \ldots$, perform the following:

(a) Construct horizon group $\mathscr{G}_{s}$ that contains $\hbar$ horizon sizes using definition (9) as follows:

$$
\begin{aligned}
\mathscr{G}_{s} & =\left\{2 \Xi_{s}^{c}-\hbar, 2 \Xi_{s}^{c}+\zeta-\hbar, \ldots, 2 \Xi_{s}^{c}+\zeta \times(\hbar-1)\right. \\
& \left.-\hbar, 2 \Xi_{s}^{c}+\zeta \times \hbar-\hbar\right\}=\left\{\Xi_{s}^{1}, \Xi_{s}^{2}, \ldots, \Xi_{s}^{i}, \ldots, \Xi_{s}^{\hbar+1}\right\} .
\end{aligned}
$$

(b) Using the horizon sizes $\Xi_{s}^{i}(i=1,2, \ldots, \hbar+1)$ contained in $\mathscr{G}_{s}$, perform the following:

$$
\begin{aligned}
& \text { for } i=1 \text { to } \hbar+1 \text { do } \\
& \text { if } \Xi_{s}^{i}<s \text { and } \widetilde{\Xi}_{\min }<\Xi_{s}^{i}<\widetilde{\Xi}_{\max } \text {; then }
\end{aligned}
$$

(i) Obtain $\hat{x}_{s}\left(\Xi_{s}^{i}\right)$ by performing DFIR estimator (Algorithm 10) using $\Xi_{s}^{i}$ as follows:

$$
\begin{aligned}
\widehat{x}_{s \mid s-1}^{[\lambda]}\left(\Xi_{s}^{i}\right)= & \left(\mathscr{F}_{\Xi_{s}^{i}+\hbar, \gamma(s)}^{T} \Theta_{\Xi_{s}^{i}+\hbar, \gamma(s)}^{-1} \mathscr{F}_{\Xi_{s}^{i}+\hbar, \gamma(s)}\right)^{-1} \\
& \cdot \mathscr{F}_{\Xi_{s}^{i}+\hbar, \gamma(s)}^{T} \Theta_{\Xi_{s}^{i}+\hbar, \gamma(s)}^{-1} \widetilde{\mathscr{Y}}_{s-1}^{[\lambda]}\left(\Xi_{s}^{i}\right),
\end{aligned}
$$

where $\mathscr{F}_{\Xi_{s}^{i}+\hbar, \gamma(s)}, \Theta_{\Xi_{s}^{i}+\hbar, \gamma(s)}$, and $\widetilde{\mathscr{Y}}_{s-1}^{[\lambda]}\left(\Xi_{s}^{i}\right)$ can be obtained using the horizon size $\Xi_{s}^{i}$ and definition (37).

(ii) Compute the likelihood of $\hat{x}_{s}\left(\Xi_{s}^{i}\right)$ using (49) as

$$
\begin{gathered}
\xi\left(\hat{x}_{s}^{i}\left(\Xi_{s}^{i}\right)\right)=\frac{1}{\sqrt{(2 \pi)^{q} \operatorname{det}(\bar{R})}} \\
\times \exp \left\{-\frac{1}{2}\left(y_{m}^{[\lambda]}(s)-\vartheta_{s}^{[\lambda]} \widetilde{H}_{m}^{[\lambda]}(s) \hat{x}_{s}^{i}\left(\Xi_{s}^{i}\right)\right)^{T}\right. \\
\left.\cdot \bar{R}^{-1}\left(y_{m}^{[\lambda]}(s)-\vartheta_{s}^{[\lambda]} \widetilde{H}_{m}^{[\lambda]}(s) \hat{x}_{s}^{i}\left(\Xi_{s}^{i}\right)\right)\right\} . \\
\text { endif }
\end{gathered}
$$$$
\text { endfor }
$$

(c) The output (i.e., state estimate) of DFIR at time $s$, denoted by $\hat{x}_{s}$, is determined as

$$
\widehat{x}_{s}=\arg \max _{\hat{x}_{s}\left(\Xi_{s}^{i}\right)} \xi\left(\hat{x}_{s}\left(\Xi_{s}^{i}\right)\right) \text {. }
$$

(d) The center size at time $s+1$, denoted by $\Xi_{s+1}^{c}$, is determined as follows:

$$
\Xi_{s+1}^{c}=\arg \max _{\Xi_{s}^{c}} \xi\left(\widehat{x}_{s}\left(\Xi_{s}^{i}\right)\right)
$$

\section{An Application to Clustered UAV Systems}

5.1. Simulation Configurations and Metrics. In this section, we illustrate the results proposed in Sections 3 and 4 by numerical simulations. The position and velocity of a generic UAV evolve according to the state-space model as follows:

$$
\begin{aligned}
{\left[\begin{array}{c}
x_{p}(t+1) \\
x_{v}(t+1)
\end{array}\right]=} & {\left[\begin{array}{cc}
I_{3} & \delta_{t} \cdot I_{3} \\
\mathbf{0}_{3 \times 3} & I_{3}
\end{array}\right]\left[\begin{array}{c}
x_{p}(t) \\
x_{v}(t)
\end{array}\right] } \\
& +\left[\begin{array}{c}
0.5 \cdot \delta_{t}^{2} \cdot \mathbf{1}_{3 \times 1} \\
\delta_{t} \cdot \mathbf{1}_{3 \times 1}
\end{array}\right] w(t),
\end{aligned}
$$

where $x_{p}(t)$ and $x_{v}(t)$ denote the position and velocity of a generic UAV at time $t$, respectively, $\delta_{t}$ represents the sampling period, and $\delta_{t}=0.1 s$; and $w(t)$ is a zero-mean white noise with variance $Q_{w}, Q_{w}=0.01 I_{6}$; the initial values are $\widehat{x}_{0 \mid 0}=$ $[120,30,0,0,0,0]^{T}$ and $P_{0 \mid 0}=\operatorname{diag}\left\{25^{2} \mathbf{I}_{3}, 0.1^{2} \mathbf{I}_{3}\right\}$.

Consider a fleet of $N_{\Sigma}=75$ UAVs deployed schematically over a field of $[-5,5] \times[-5,5] \times[0,1] \mathrm{km}$. The initial network topology is depicted in Figure 3(a). The objective UAV is set to track a trajectory as in Figure 3(b) and in flight for $1000 \mathrm{sec}$.

The objective UAV updates its state essentially based on cooperative packets of its neighbors, which include the time stamp $s$ and the navigational state of the sender $\hat{x}_{t}^{[i]}=$

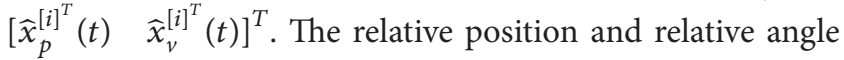
are measured by RSS and AOA methods, respectively, with the observation models as in (4).

For RSS measurements, the transmission power is taken as $K=20 \mathrm{dBm}$, the path loss exponent is $\lambda=2.1$, and $\alpha=$ 32.44. The standard deviation for measurement noise of AOA is 0.1 degrees. The variances of LOS and NLOS measurement noises are taken as $R_{t, 1}^{[i]}=64$ and $R_{t, 2}^{[i]}=100$, respectively. The bias of the NLOS measurement error is assumed to be $\mu_{t}^{[i]}=-5$. The occurrence probability of NLOS measurement error is taken as $P_{\text {NLOS }}=0.5$ for each neighbor. The transition probabilities of Markov chain $\lambda_{t}^{[i]}$ are set as $\pi_{11}^{(2)}=\pi_{22}^{(2)}=0.9$ and $\pi_{12}^{(2)}=\pi_{21}^{(2)}=0.1$. To reduce the computational cost, not all the cooperative measurements are used; instead, only $\ell=$ 5 strongest received signals are adopted for state update of the objective UAV.

As to the random delay, it is assumed that $\tau(t) \epsilon$ $\{0,1,2,3\} . \tau(t)$ is a prior known to the estimator and its paths can be described by $\kappa_{t, \tau}^{[i]}(i=1,2, \ldots, \ell)$ with the restriction $\kappa_{t+\tau_{1}, \tau_{1}}^{[i]} \times \kappa_{t+\tau_{2}, \tau_{2}}^{[i]}=0$ if $\tau_{1} \neq \tau_{2}$. Hereinto, it is assumed that $\tau(t)$ is of the probabilities

$$
\begin{aligned}
& \operatorname{Prob}\{\tau(t)=0\}=0.4, \\
& \operatorname{Prob}\{\tau(t)=1\}=0.3, \\
& \operatorname{Prob}\{\tau(t)=2\}=0.2, \\
& \operatorname{Prob}\{\tau(t)=3\}=0.1
\end{aligned}
$$

A distributed fusion receding horizon estimator (DRHE) in [25] is adopted to compare with DFIR in Section 3 and the AFGE in Section 4. Let RMSE denote the root mean square error for the estimation of $x_{t}^{[i]}$; that is, $\left((1 / \mathscr{M}) \sum_{j=1}^{M}\left(x_{t}^{[j]}-\right.\right.$ $\left.\left.\widehat{x}_{t \mid t}^{[j]}\right)^{2}\right)^{0.5}$, where $\mathscr{M}=500$ is the Monte Carlo simulation runs.

5.2. Simulation Results and Analysis. In this subsection, several key properties of the proposed AFGE approach, that 


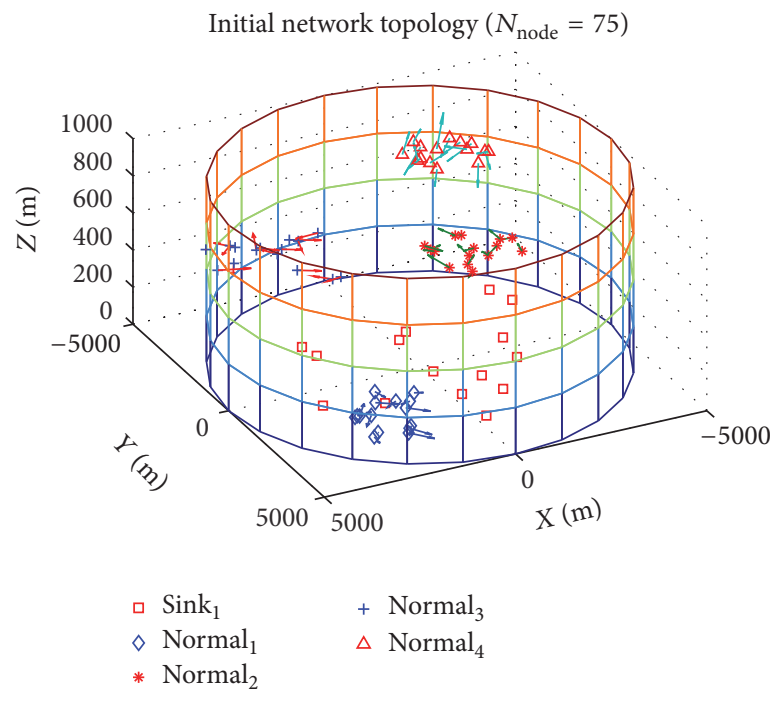

(a)

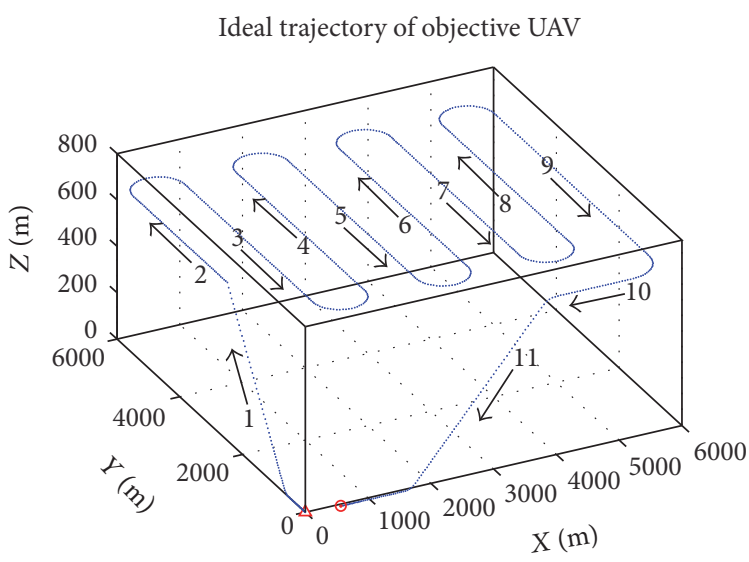

(b)

FIGURE 3: Initial settings of network topology and ideal trajectory. (a) Initial topology of the networked vehicles. (b) Ideal trajectory of objective vehicle.

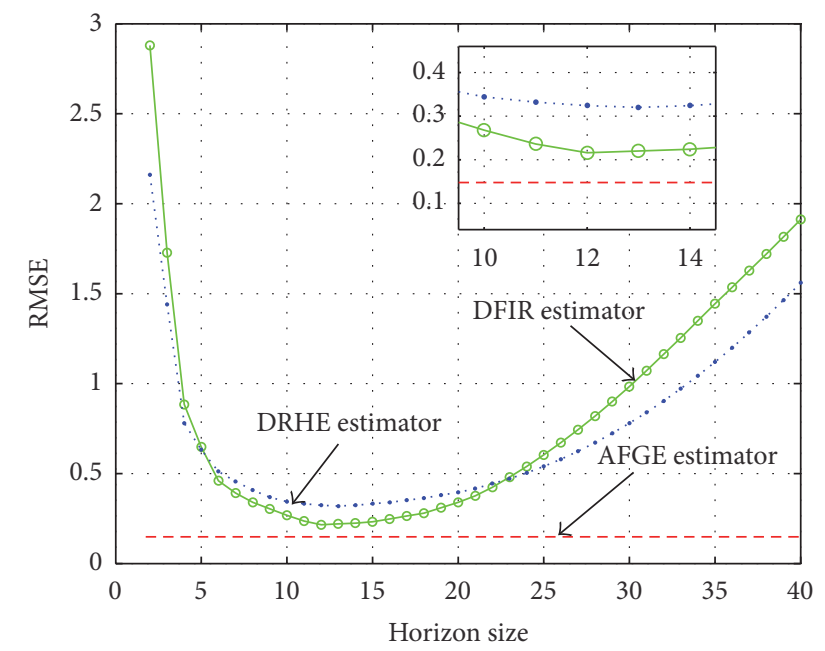

FIGURE 4: RMSEs comparison of DFIR, DRHE, and AFGE estimators.

is, AHG scheme and estimation errors, are tested on the networked UAVs with the models in Section 5.1. The design parameters of the AFGE algorithm are taken as $\hbar=3, \zeta=1$, $\Xi_{\text {min }}=2$, and $\Xi_{\max }=40$.

First, we verify that AHG improves the estimation performance of the DFIR estimator by adapting the horizon size. To this end, we compare the AFGE with DFIR and DRHE. While the DFIR uses a constant horizon size, the AFGE uses the adaptive horizon size. Figure 4 depicts the RMSE comparisons among DFIR, DRHE, and AFGE for diverse horizon sizes in the rectilinear orbit determination problem. In addition, this figure shows the RMSE of the AFGE estimator. The DFIR estimator produces the minimum RMSE of 0.216 when the horizon size is 12 , whereas the minimum RMSE of DRHE is 0.320 , with the horizon size of
13. The RMSE of the AFGE is 0.196, which is smaller than that of DFIR and DRHE. AFGE outperforms the DFIR using the best constant horizon size. Thus, it is verified that AHG improves the estimation performance of the DFIR.

Next, we consider the UAV navigation scenario with the initial settings as in Section 5.1. Besides, we set the horizon size of DFIR to $\Xi^{[\lambda]}=12$, according to the analysis in Figure 4. The simulation results can be found in Figures 5 and 6.

Hereinto, Figure 5(a) reports the localization results based on RSS/AOA relative measurement and the CPs of its neighbors, while the related parameters are recorded in Figures 5(b) and 5(c). It is clear from Figure 5(b) that the number of received CPs in each step is greater than four, which means the observations are sufficient for the geometric localization in three-dimension space [9]. One path of $\tau(t)$ is given in Figure 5(c), based on which the optimal filter can be obtained directly by using the scheme in Sections 3 and 4. As expected, the localization result in Figure 5(a) demonstrates the validity of the proposed AFGE algorithm and navigation scheme.

More specifically, Figures 6(a) and 6(b) present the comparison of estimation results on location and velocity errors, respectively. The estimators of DFIR in Section 3 and DRHE [25] are chosen as the comparison with our proposed AFGE algorithm.

As shown in the simulation results, the convergence rate of the DRHE is faster than that of DFIR due to the fact that DRHE adopts a distributed fusion structure in the design of estimator; however, the convergence error of the DFIR is smaller than that of DRHE, which is partly due to its reorganization of delayed observations and is also due to the design of DFIR considering the Markov jumping of LOS/NLOS errors. Furthermore, the performance of AFGE outperforms DRHE and DFIR; the reason can be owed to its reorganization scheme for delayed observation on one hand 


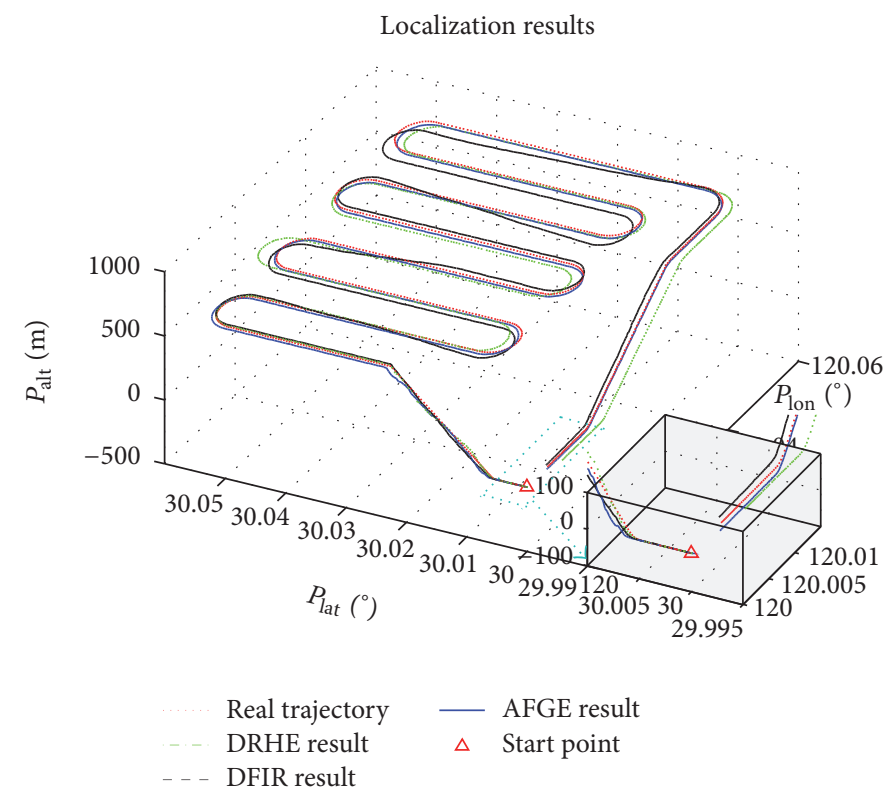

(a)

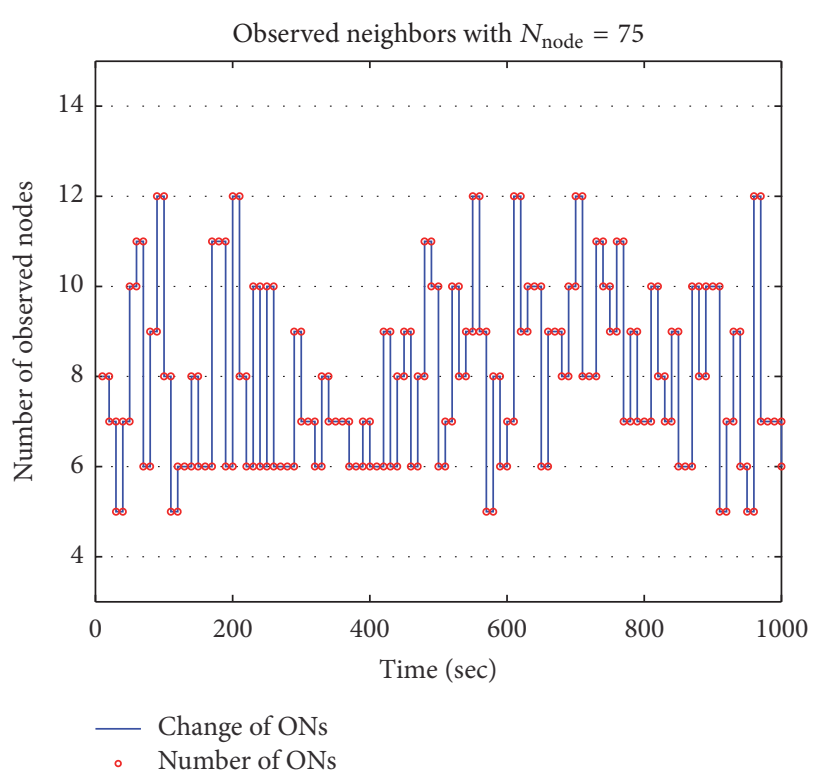

(b)
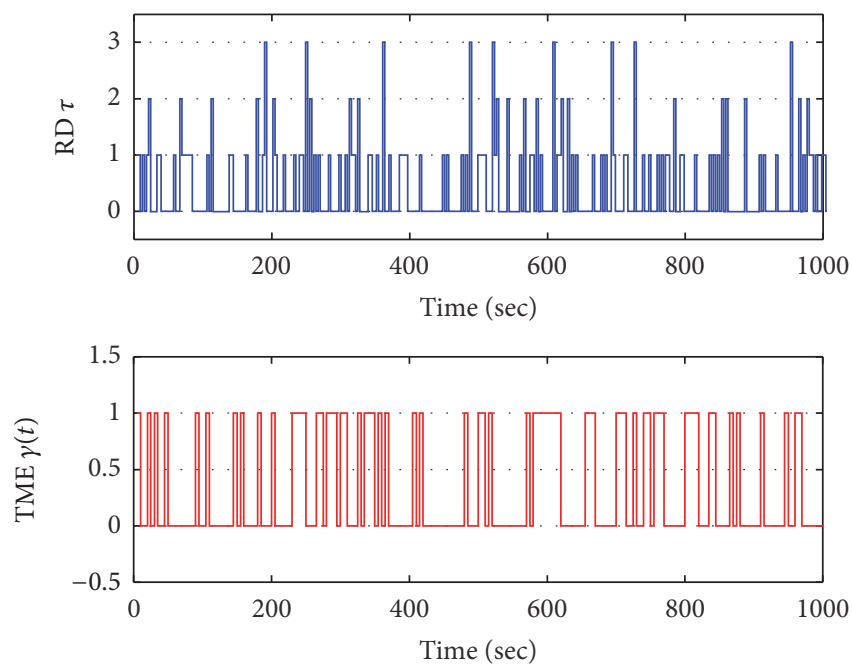

(c)

FIGURE 5: Localization results comparison and the related parameters. (a) Localization trajectories based on DRHE, DFIR, and AFGE. (b) Number of corresponding observable neighbors. (c) Random delay $\tau(t) \in\{0,1,2,3\}$ and the transition of NOS/NLOS errors $\gamma(t) \in\{0,1\}$.

and considering the Markov jumping of LOS/NLOS errors on the other hand. Besides, the proposed AFGE also benefits from the AHG policy, which can manage the horizon size, thus acquiring the fastest convergence rate with the smallest estimation error.

\section{Conclusions and Discussions}

In this paper, an adaptive horizon estimator has been addressed to solve the state estimation of NNS with the features of remote sensing complementary observations (RSOs) under mixed LOS/NLOS environments. An adaptive finitehorizon group estimator (AFGE) has been proposed, where the horizon size can be adjusted in real time according to stochastic parameters and random delays. First, a delay-aware FIR (DFIR) estimator has been derived with observation reorganization and complementary fusion strategies. Second, an adaptive horizon group (AHG) policy has been proposed to manage the horizon size. The AFGE algorithm is thus realized by combining AHG policy and DFIR estimator. A numerical simulation example has been used to demonstrate the potential of the proposed approach to robust estimation in terms of both performances and computational tractability.

Future research will focus on other typical issues of NNS in terms of multirate fusion problem and cooperative attitude estimation problem. 

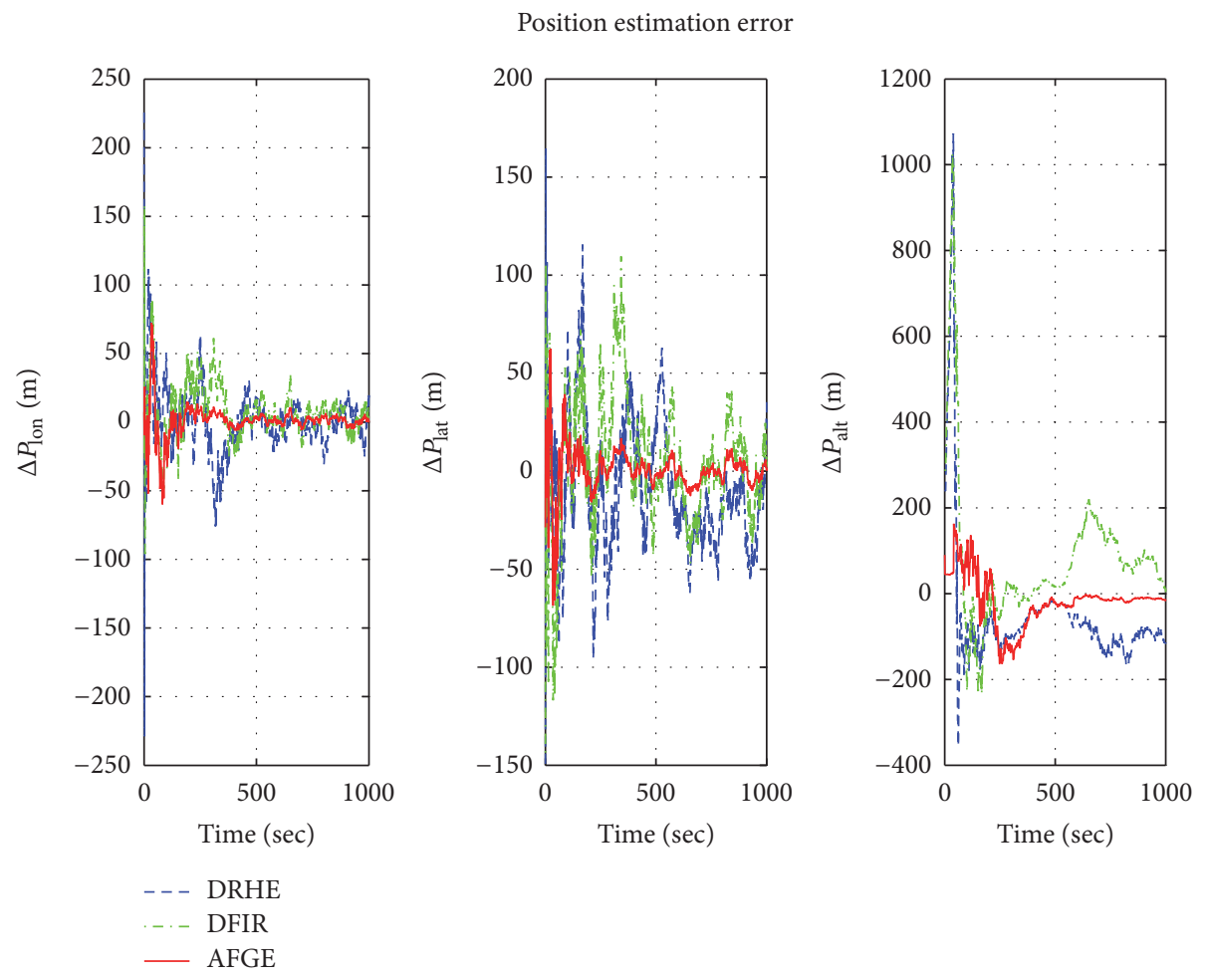

-.. DFIR

(a)

Velocity estimation error
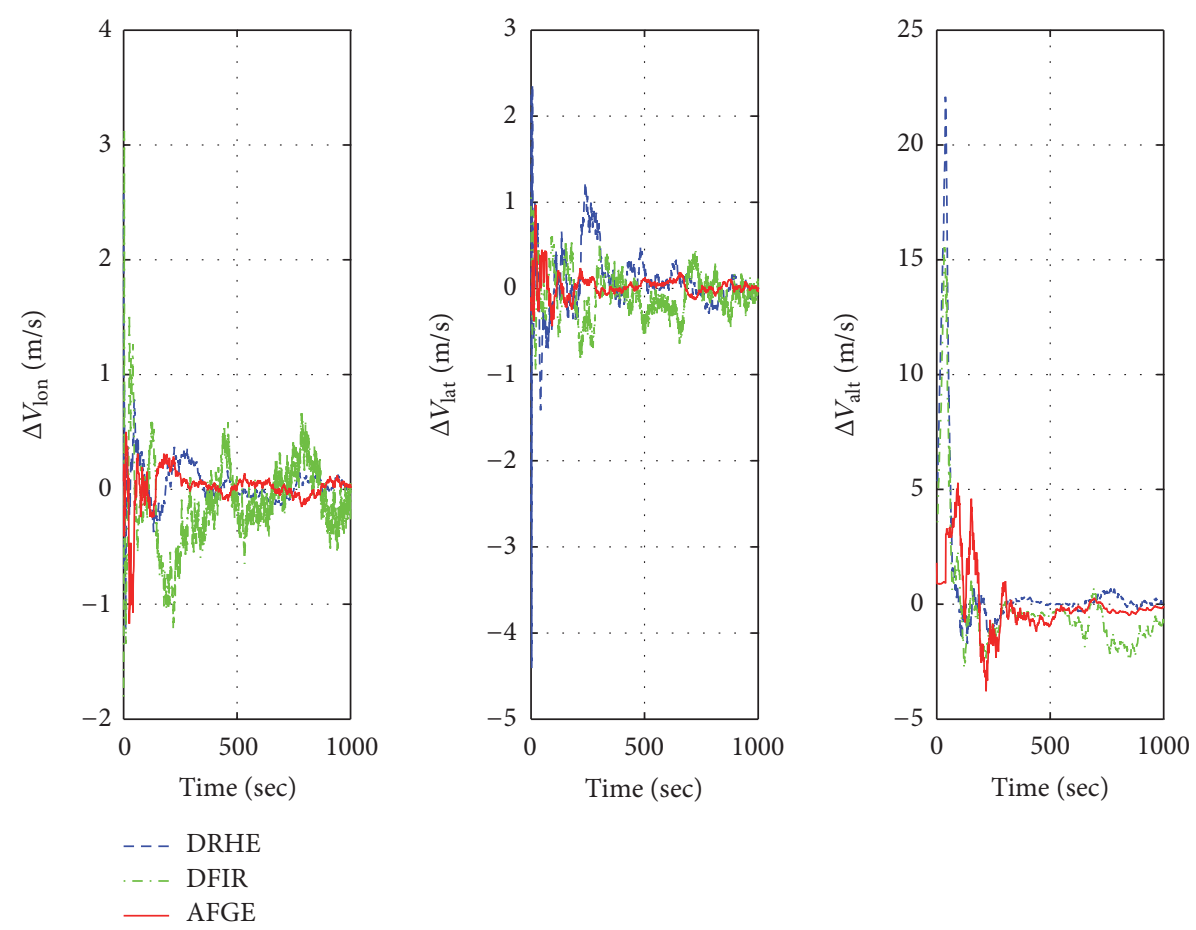

(b)

FIGURE 6: Performance comparison of estimation results between DRHE, DFIR, and AFGE. (a) Localization results. (b) Velocity results. 


\section{Competing Interests}

The authors declare that they have no competing interests.

\section{Acknowledgments}

This work was supported in part by the National Natural Science Foundation of China under Grant nos. 61473306 and 61203168 and partly by the Defense Advanced Research Project of China under Grant no. 9140A09040614JB14001.

\section{References}

[1] C. Gao, G. Zhao, J. Lu, and S. Pan, "Decentralised movinghorizon state estimation for a class of networked spatialnavigation systems with random parametric uncertainties and communication link failures," IET Control Theory \& Applications, vol. 9, no. 18, pp. 2666-2677, 2015.

[2] C. Gao, G. R. Zhao, J. H. Lu, and S. Pan, "A grid-based cooperative QoS routing protocol with fading memory optimization for navigation carrier ad hoc networks," Computer Networks, vol. 76, pp. 294-316, 2015.

[3] B. Allotta, R. Costanzi, E. Meli, L. Pugi, A. Ridolfi, and G. Vettori, "Cooperative localization of a team of AUVs by a tetrahedral configuration," Robotics and Autonomous Systems, vol. 62, no. 8, pp. 1228-1237, 2014.

[4] M. Bibuli, G. Bruzzone, M. Caccia, A. Ranieri, and E. Zereik, "Multi-vehicle cooperative path-following guidance system for diver operation support," in Proceedings of the 10th IFAC Conference on Manoeuvring and Control of Marine Craft (MCMC '15), pp. 75-80, Copenhagen, Denmark, August 2015.

[5] Z. X. Jiang and B. T. Cui, "Estimation of spatially distributed processes using mobile sensor networks with missing measurements," Chinese Physics B, vol. 24, no. 2, Article ID 020702, 2015.

[6] C. Toth and G. Józków, "Remote sensing platforms and sensors: a survey," ISPRS Journal of Photogrammetry and Remote Sensing, vol. 115, pp. 22-36, 2016.

[7] S. M. Azizi and K. Khorasani, "Networked estimation of relative sensing multiagent systems using reconfigurable sets of subobservers," IEEE Transactions on Control Systems Technology, vol. 22, no. 6, pp. 2188-2204, 2014.

[8] T. A. Wettergren, "Performance of search via track-beforedetect for distributed sensor networks," IEEE Transactions on Aerospace and Electronic Systems, vol. 44, no. 1, pp. 314-325, 2008.

[9] A. F. H. Goetz, J. B. Wellman, and W. L. Barnes, "Optical remote sensing of the earth," Proceedings of the IEEE, vol. 73, no. 6, pp. 950-969, 1985.

[10] F.-Q. Wen, G. Zhang, and D. Ben, "Direction-of-arrival estimation for co-located multiple-input multiple-output radar using structural sparsity Bayesian learning," Chinese Physics B, vol. 24, no. 11, Article ID 110201, 2015.

[11] I. Güvenç and C.-C. Chong, "A survey on TOA based wireless localization and NLOS mitigation techniques," IEEE Communications Surveys and Tutorials, vol. 11, no. 3, pp. 107-124, 2009.

[12] K. Yu and Y. J. Guo, "Statistical NLOS identification based on AOA, TOA, and signal strength," IEEE Transactions on Vehicular Technology, vol. 58, no. 1, pp. 274-286, 2009.

[13] M. Heidari, N. A. Alsindi, and K. Pahlavan, "UDP identification and error mitigation in ToA-based indoor localization systems using neural network architecture," IEEE Transactions on Wireless Communications, vol. 8, no. 7, pp. 3597-3607, 2009.

[14] F. Yin, C. Fritsche, F. Gustafsson, and A. M. Zoubir, "EMand JMAP-ML based joint estimation algorithms for robust wireless geolocation in mixed LOS/NLOS environments," IEEE Transactions on Signal Processing, vol. 62, no. 1, pp. 168-182, 2014.

[15] C. Morelli, M. Nicoli, V. Rampa, and U. Spagnolini, "Hidden Markov models for radio localization in mixed LOS/NLOS conditions," IEEE Transactions on Signal Processing, vol. 55, no. 4, pp. 1525-1542, 2007.

[16] L. Yi, S. G. Razul, Z. Lin, and C. M. See, "Individual AOA measurement detection algorithm for target tracking in mixed LOS/NLOS environments," in Proceedings of the IEEE International Conference on Acoustics, Speech and Signal Processing (ICASSP '13), pp. 3924-3928, Vancouver, Canada, May 2013.

[17] L. Yi, S. G. Razul, Z. Lin, and C. M. See, "Target tracking in mixed LOS/NLOS environments based on individual measurement estimation and LOS detection," IEEE Transactions on Wireless Communications, vol. 13, no. 1, pp. 99-111, 2014.

[18] M. C. Vanderveen, C. B. Papadias, and A. Paulraj, "Joint angle and delay estimation (JADE) for multipath signals arriving at an antenna array," IEEE Communications Letters, vol. 1, no. 1, pp. 12-14, 1997.

[19] J. H. Lu, C. Gao, G. R. Zhao, and S. Liu, "Decentralized state estimation for networked navigation systems with communication delay and packet loss: the receding horizon case," in Proceedings of the 17th IFAC Symposium on System Identification, pp. 10941099, Beijing, China, October 2015.

[20] A. M. Syed and R. Saravanakumar, "Augmented Lyapunov approach to $H_{\infty}$ state estimation of static neural networks with discrete and distributed time-varying delays," Chinese Physics $B$, vol. 24, Article ID 050201, 2015.

[21] C. Gao, G. R. Zhao, S. Liu, and J. H. Lu, "Decentralized navigational state estimation for networked multiple vehicles subject to limited data rate," in Proceedings of the 17th IFAC Symposium on System Identification, pp. 1088-1093, Beijing, China, October 2015.

[22] B. Q. Xue, S. Y. Li, and Q. M. Zhu, "Moving horizon state estimation for networked control system with multiple packet dropouts," IEEE Transactions on Automatic Control, vol. 57, no. 9, pp. 2360-2366, 2012.

[23] G. C. Goodwin, H. Haimovich, D. E. Quevedo, and J. S. Welsh, "A moving horizon approach to networked control system design," IEEE Transactions on Automatic Control, vol. 49, no. 9, pp. 1427-1445, 2004.

[24] W. H. Kwon, P. S. Kim, and P. Park, "A receding horizon Kalman FIR filter for discrete time-invariant systems," IEEE Transactions on Automatic Control, vol. 44, no. 9, pp. 1787-1791, 1999.

[25] I. Y. Song and V. Shin, "Distributed mixed continuous-discrete receding horizon filter for multisensory uncertain active suspension systems with measurement delays," IET Control Theory \& Applications, vol. 7, no. 15, pp. 1922-1931, 2013.

[26] J. M. Pak, C. K. Ahn, C. J. Lee, P. Shi, M. T. Lim, and M. K. Song, "Fuzzy horizon group shift FIR filtering for nonlinear systems with Takagi-Sugeno model," Neurocomputing, vol. 174, pp. 10131020, 2016. 


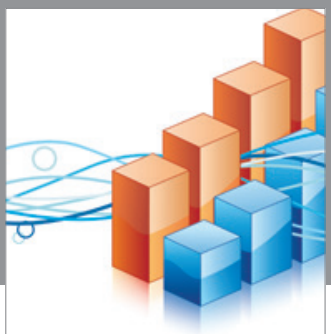

Advances in

Operations Research

vatem alat4

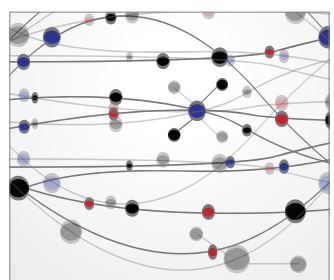

\section{The Scientific} World Journal
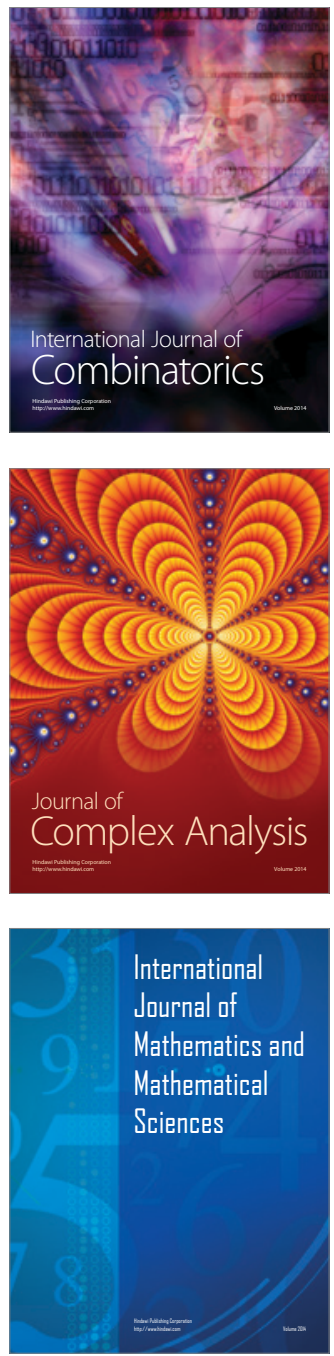
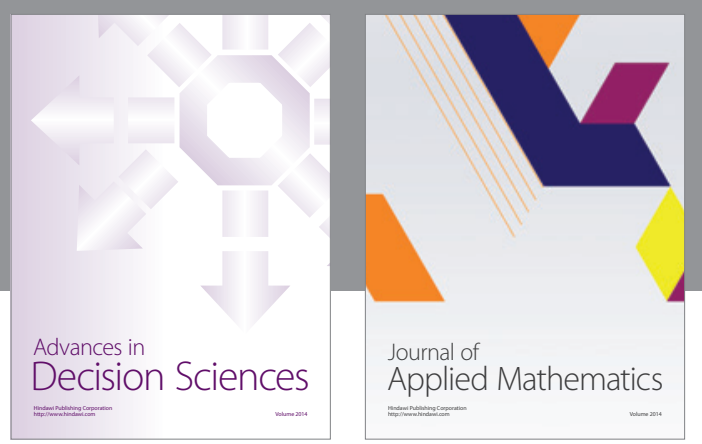

Algebra

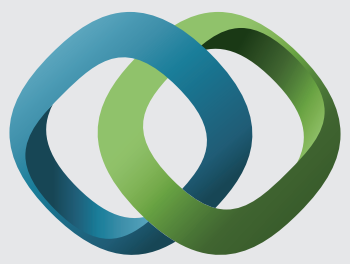

\section{Hindawi}

Submit your manuscripts at

http://www.hindawi.com
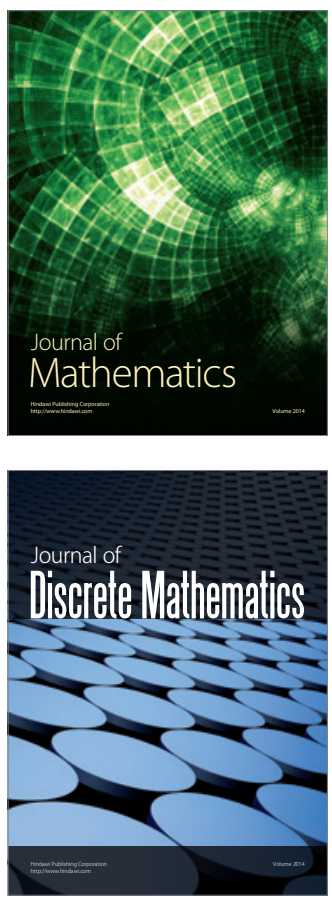

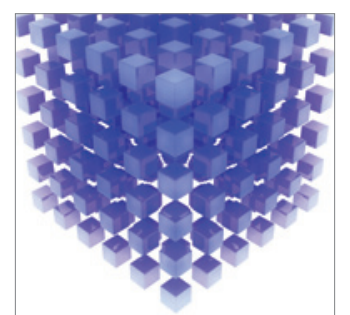

Mathematical Problems in Engineering
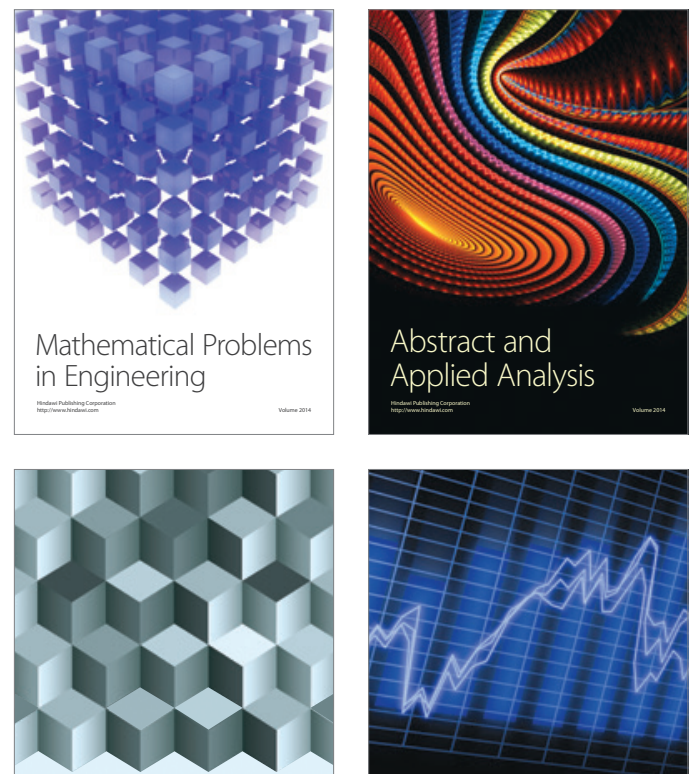

Journal of

Function Spaces

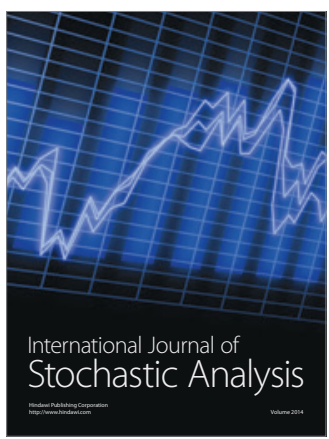

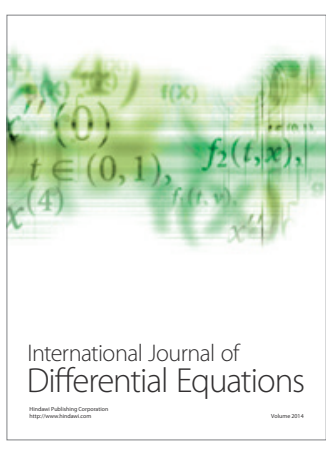
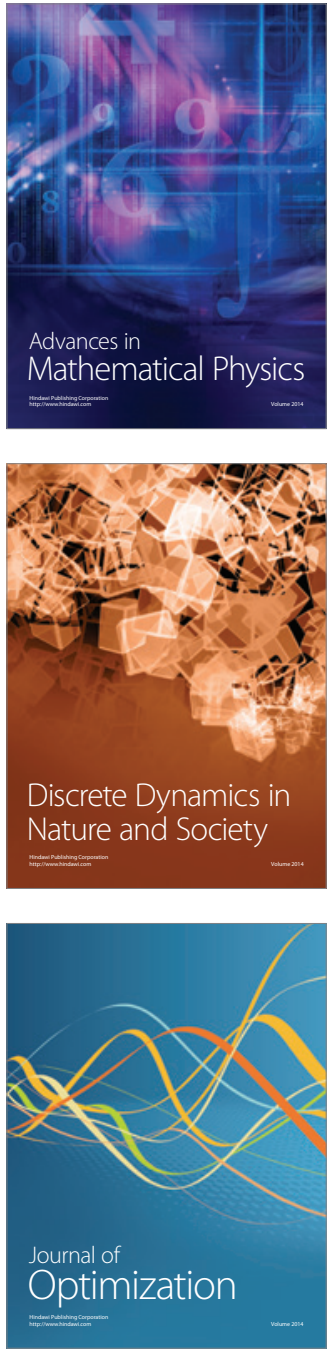